\title{
Flight Test of Orthogonal Square Wave Inputs for Hybrid-Wing-Body Parameter Estimation
}

\author{
Brian R. Taylor ${ }^{1}$ and Nalin A. Ratnayake ${ }^{2}$ \\ NASA Dryden Flight Research Center, Edwards, California, 93523
}

\begin{abstract}
As part of an effort to improve emissions, noise, and performance of next generation aircraft, it is expected that future aircraft will use distributed, multi-objective control effectors in a closed-loop flight control system. Correlation challenges associated with parameter estimation will arise with this expected aircraft configuration. The research presented in this paper focuses on addressing the correlation problem with an appropriate input design technique in order to determine individual control surface effectiveness. This technique was validated through flight-testing an 8.5-percent-scale hybrid-wing-body aircraft demonstrator at the NASA Dryden Flight Research Center (Edwards, California). An input design technique that uses mutually orthogonal square wave inputs for de-correlation of control surfaces is proposed. Flight-test results are compared with prior flight-test results for a different maneuver style.
\end{abstract}

\section{Introduction}

$\mathrm{E}^{\mathrm{n}}$ nvironmentally Responsible Aviation (ERA) is a National Aeronautics and Space Administration (NASA) project designed to improve emissions, noise, and performance of next generation aircraft. ${ }^{1}$ It is expected that many of these aircraft will make use of redundant, multi-objective control effectors in a closed-loop flight control system. A distributed control system can tailor the wing to optimize performance at multiple operating points and provides redundancy for control surface failure. Isolating the aircraft response due to each control surface becomes difficult with large numbers of nearly coplanar control surfaces operating in a closed-loop flight control system. ${ }^{2}$ Due to correlation problems, analysis of these aircraft will require advances in parameter estimation tools and methods to enable validation of aerodynamic models, simulations, and control laws.

Estimation of single surface control effectiveness for aircraft with many multi-objective, redundant control surfaces is possible with appropriate input design methods. Each surface capable of contributing to the aircraft response must be excited and de-correlated. Prior research has involved using aerodynamic models to reduce the number of parameters being estimated in order to de-correlate inputs. ${ }^{3}$ Other research has used time separation to de-correlate inputs ${ }^{2}$ or sine waves that are mutually orthogonal in the frequency domain. ${ }^{4}$ This paper presents an approach that uses square wave inputs that are mutually orthogonal in the time domain.

Longitudinal parameter estimation results are disseminated in this paper for the orthogonal square wave inputs. Flight-test data was used to estimate control surface effectiveness for a range of angles of attack, which was compared against prior flight estimates that used temporal separation for de-correlation. Only control effectiveness estimates are shown in this paper because rigid body parameters can be estimated with more traditional techniques. Additionally, mutually orthogonal square wave inputs keep the aircraft near trim, which is desired for control effectiveness estimates, but may not produce enough motion to estimate the lift- or drag-based parameters accurately.

\section{Aircraft Description}

The X-48B aircraft, ${ }^{5}$ shown in Fig. 1, is a joint partnership between NASA, the Air Force Research Laboratory (AFRL), and The Boeing Company (Chicago, Illinois). Built by Cranfield Aerospace Limited (United Kingdom), the aircraft is a remotely-piloted, 8.5-percent dynamically scaled hybrid-wing-body (HWB) aircraft with twenty aerodynamic control surfaces and three JetCat (JetCat USA, Paso Robles, California) turbojet engines. Eighteen of

\footnotetext{
${ }^{1}$ Aerospace Engineer, Controls and Dynamics Branch, P.O. Box 273, MS 4840D, AIAA Member.

${ }^{2}$ Aerospace Engineer, Aerodynamics and Propulsion Branch, P.O. Box 273, MS 4840B, AIAA Senior Member. 
the twenty control surfaces are redundant and multi-objective. Allocation of the control surfaces is depicted in Fig. 2 with surface pairs numbered for reference. Additionally, surface 6 and surface 7 are split ailerons: the top and bottom surface can be moved together to produce roll moments or they can be split to produce a yaw moment. Rudders are also incorporated into the winglets to provide yaw control.

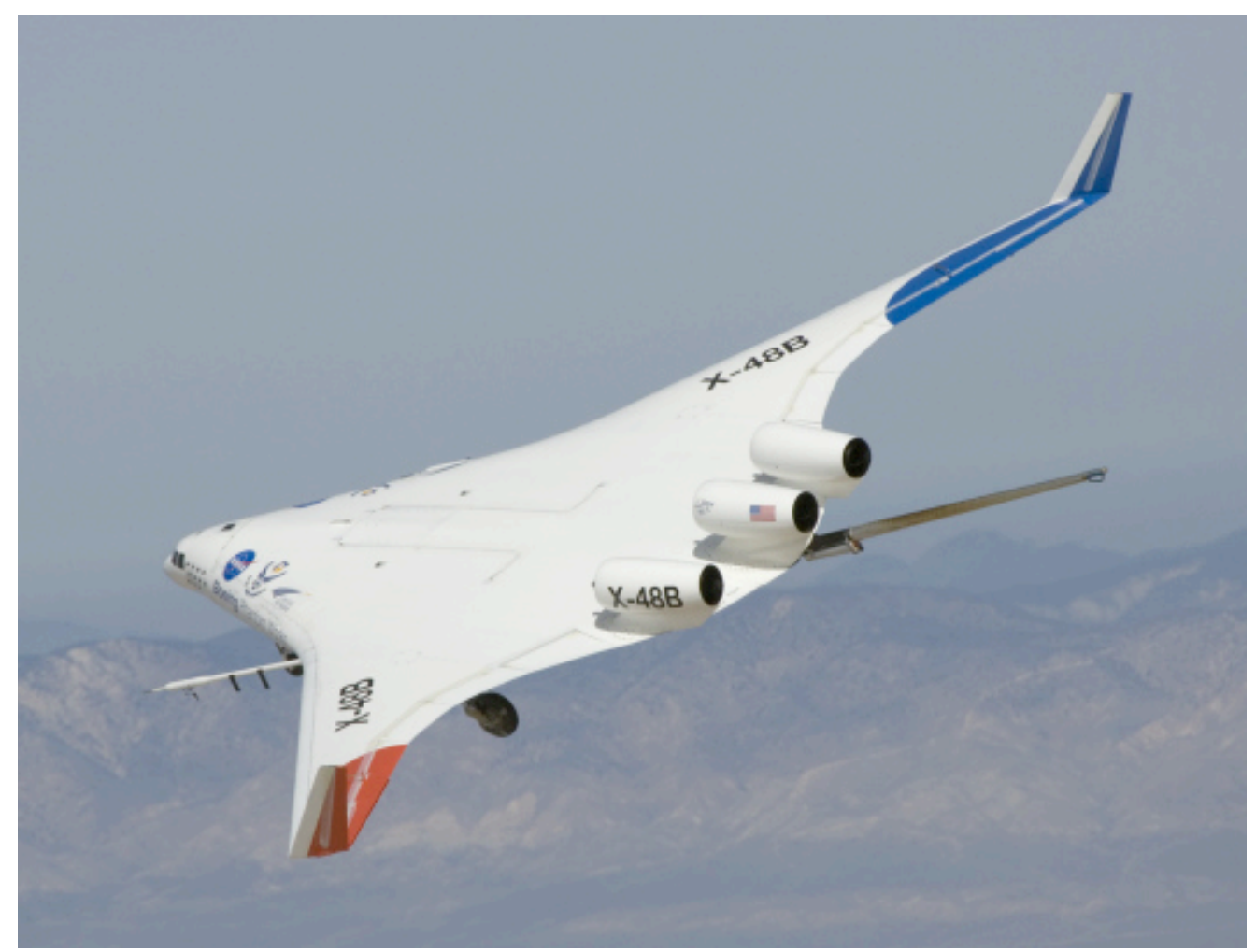

Figure 1. The $\mathrm{X}-48 \mathrm{~B}$ aircraft in flight.

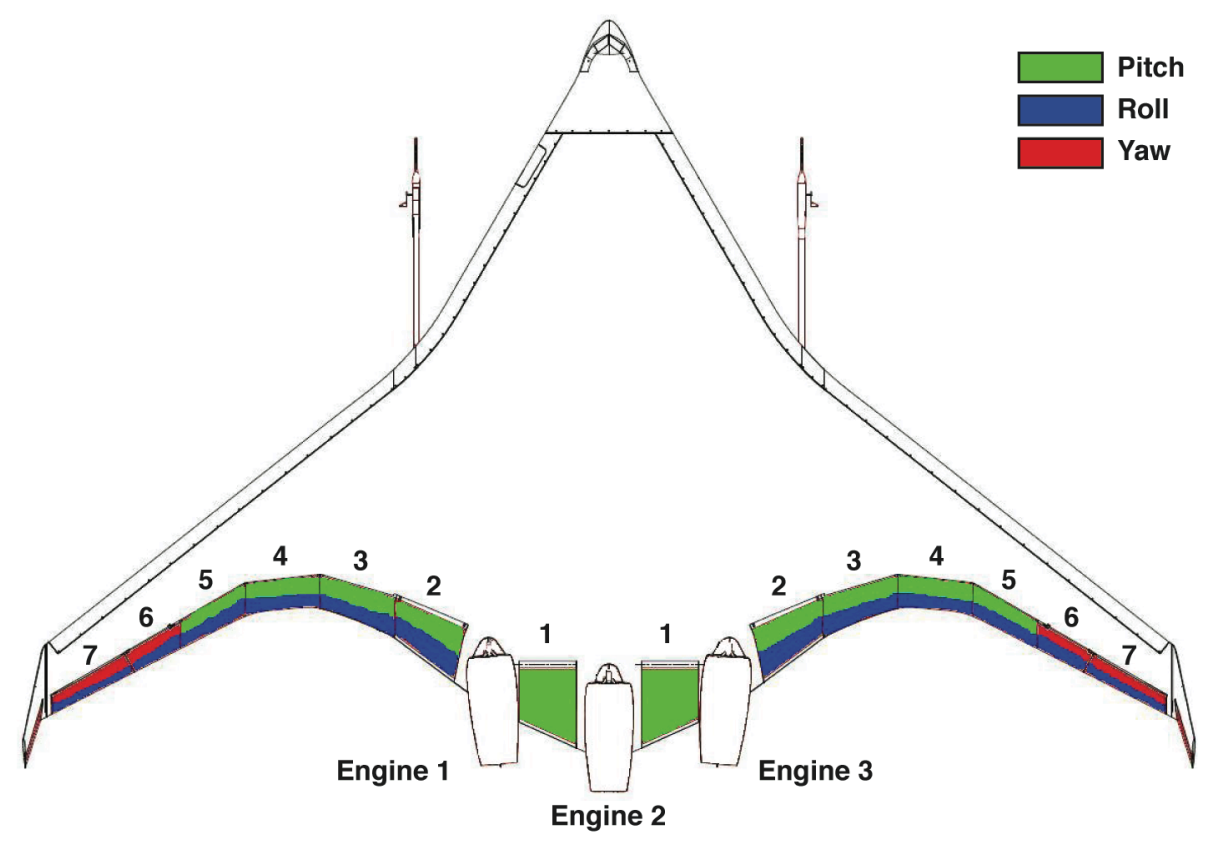

Figure 2. The control surface allocation of the $\mathrm{X}-48 \mathrm{~B}$.

American Institute of Aeronautics and Astronautics 
The X-48B uses an On Board Excitation System (OBES) for exciting the control surfaces for parameter estimation. The OBES has the ability to input waveforms directly on the individual surface commands, which are added to the commands from the closed-loop controller.

Due to the inherent longitudinal instability of the aircraft in parts of the flight envelope, it is augmented with a closed-loop flight control system. At the flight conditions used for parameter estimation in this paper, the aircraft was statically stable, so no changes were made to the parameter estimation algorithms used.

Instruments relevant to parameter estimation include dual airdata probes to measure airspeed, angle of attack, and angle of sideslip. Additionally, the aircraft is equipped with an inertial measurement unit / global positioning system that provides linear acceleration, angular rotation rates, and Euler angles. Control surface positions are inferred from the measured actuator position and are not measured directly. The control surface actuation on the $\mathrm{X}-48 \mathrm{~B}$ aircraft consists of an electro-mechanical servo that moves the control surface through a linkage. Position measurement is taken at the output shaft of the servo, so differences between the surface position and actuator position may be due to linkage bending or gear slop. No corrections were made to the control surface data because data or models necessary for corrections were not available. Figure 3 is a depiction of the X-48B aircraft systems and sensors. ${ }^{6}$

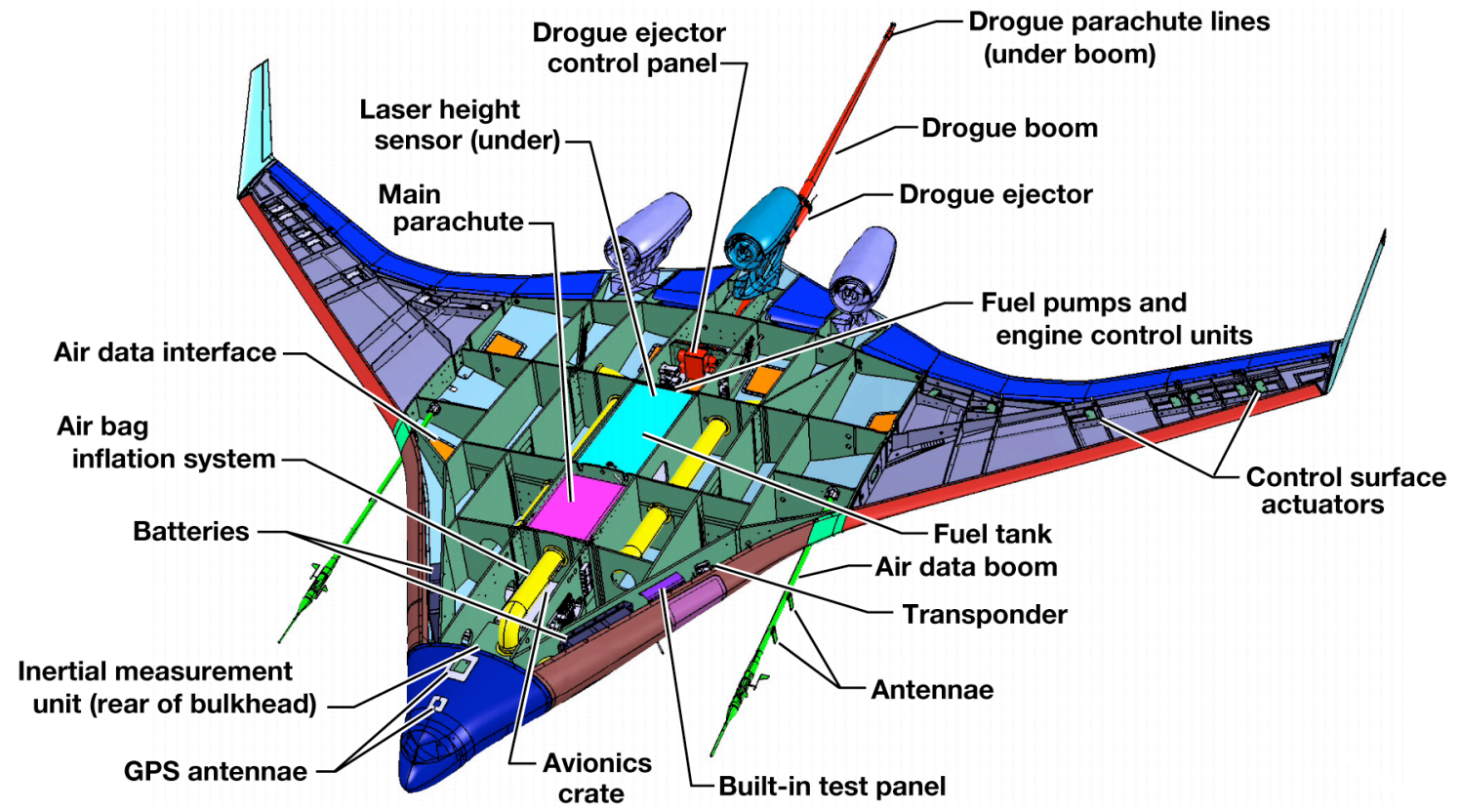

Figure 3. Systems description of the $X-48 B$ aircraft.

The aircraft can be configured with leading edge slats extended or retracted, although they cannot be adjusted in flight. The center of gravity can be adjusted on the ground between forward and aft configurations. The analysis in this paper is for the aircraft in a slats-retracted, forward center of gravity configuration.

\section{Parameter Estimation}

Parameter estimation involves the creation of mathematical models for physical systems from knowledge of the input and response of the system. As it relates to aircraft, inputs are generally control effectors, and responses are sensor measurements. Standard time-domain output-error ${ }^{7-11}$ techniques were employed for parameter estimation of both the temporal separation and square wave inputs. Output-error minimizes the difference between computed measurements and actual measurements to estimate parameters. Linear regression is a technique in which the coefficients of an assumed linear relationship between known inputs and observed outputs are estimated using least-squared fits. Linear regression was used only for the temporal separation inputs.

The Cramér-Rao lower bound represents the lowest magnitude limit for the variance of an estimator with a given bias. ${ }^{12}$ Cramér-Rao bounds were corrected for colored residuals ${ }^{5}$ and are depicted in the results as error bars on the output-error results. 


\section{Input Design Methods}

In order to determine a mathematical model for the system, the inputs to a system that cause a response must be uniquely identifiable. Challenges arise with parameter estimation of HWB aircraft due to their use of redundant, multi-objective control surfaces with highly-augmented closed-loop flight control systems. The majority of these challenges involve identifying the response attributable to each input. Redundant, multi-objective, nearly co-planar control surfaces will evoke similar aircraft responses and excite the aircraft in multiple axes. Compounding the problem, a closed-loop control system will treat all input excitations as a disturbance and attempt to damp them out with the use of the remaining control surfaces, as shown in Fig. 4. Notice that for a longitudinal maneuver, as surface pair 1 is excited, the other surface pairs that can contribute to the aircraft response move to counteract the excitation. This is in agreement with the control allocation scheme of the X-48B as shown in Fig. 2. Estimation of control effectiveness is challenging because it becomes difficult to determine the portion of the response attributable to excited surfaces.

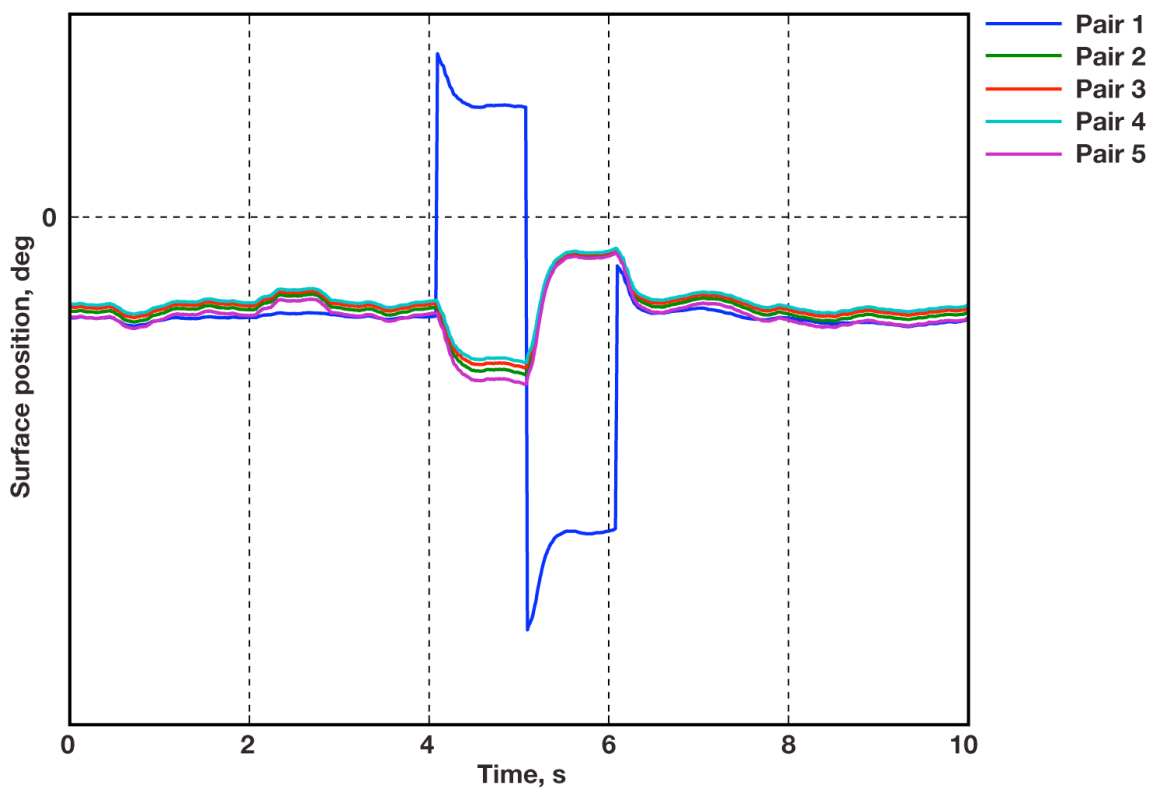

Figure 4. Input correlation.

The least accurate method for de-correlation involves using an aerodynamic model such that only the parameters corresponding to the excited input are estimated. For the example in Fig. 4, this method would use an aerodynamic model for surface pairs 2 through 5 and only estimate the effectiveness for surface pair 1. This method for de-correlation was used on the F-15 airplane (McDonnell Douglas, now The Boeing Company), but can be subject to inaccuracies caused by errors in the aerodynamic model. ${ }^{3}$

Previous research on the X-48B aircraft used temporal separation for de-correlation and estimation of control surface effectiveness. ${ }^{2}$ Each surface capable of contributing to the closed-loop response was excited individually by a doublet input and separated by time, as shown in Fig. 5. While seemingly accurate estimates were produced, it was desirable to research input design techniques that would require less flight time. 


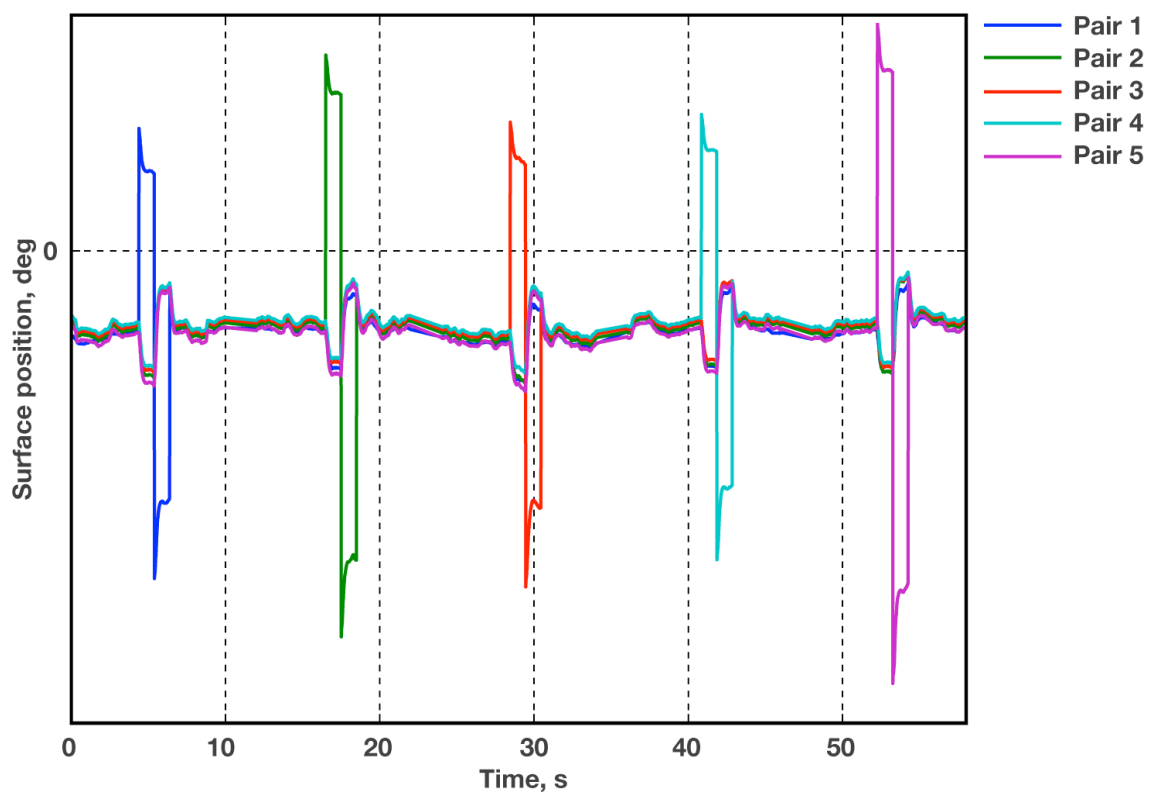

Figure 5. Temporal separation for de-correlation.

The use of orthogonal waveforms for de-correlation was a promising solution to de-correlation that would require less flight time than temporal separation. This proposed solution excites all of the surface pairs that can contribute to the aircraft response, surface pairs 1 through 5 for a longitudinal maneuver, simultaneously using orthogonal square wave inputs. While similar to multi-sine inputs, mutually orthogonal square waves were chosen because of the planned use of time domain parameter estimation techniques. Previous efforts in this area include a study of optimized inputs based on Cramér-Rao bound estimates ${ }^{13}$ using white and colored residuals. Additional research on de-correlation can be found in Refs. 14 through 19.

\section{Waveform Design}

The Hadamard matrix is a square matrix with entries that are either +1 or -1 and mutually orthogonal rows. The Hadamard matrix was used to create the parameter estimation inputs by generating a 1024 by 1024 matrix. The rows were then sampled at $200 \mathrm{~Hz}$ to create 5.12-s maneuver inputs. Frequency sweep data was analyzed to determine that the aircraft responds most to inputs around $1 \mathrm{~Hz}$. The maneuver inputs from the Hadamard matrix were not at a constant frequency; however, the average frequency of the excitation was estimated and rows that corresponded to the desired excitation frequency were chosen and repeated to make the total maneuver length $60 \mathrm{~s}$. The frequencies of adjacent surfaces were varied slightly to further de-correlate inputs. Seven different sets of frequencies were chosen to determine if any conclusions could be drawn about the frequency used, as shown in table 1 . The waveform set numbers will be used to refer to sets of frequencies throughout the remainder of this paper.

Table 1. Orthogonal waveform definitions.

\begin{tabular}{cccc}
\hline \hline & \multicolumn{3}{c}{ Frequency, Hz } \\
\hline Waveform & Surface pairs 1, 4 & Surface pairs 2, 5 & Surface pair 3 \\
\hline 41 & 0.5 & 0.25 & 0.75 \\
42 & 1.0 & 0.75 & 1.25 \\
43 & 1.5 & 1.25 & 1.75 \\
44 & 2.0 & 1.75 & 2.25 \\
45 & 1.0 & 0.5 & 1.5 \\
46 & 1.5 & 1.0 & 2.0 \\
47 & 1.5 & 0.5 & 2.5 \\
\hline \hline
\end{tabular}

American Institute of Aeronautics and Astronautics 
Figure 6a depicts the input for surface pairs 1 and 4; Fig. $6 \mathrm{~b}$ depicts surface pairs 2 and 5; and Fig. 6c depicts surface pair 3. Notice that while the square waves in Fig. 6a are not all at a constant frequency, they are close to $0.5 \mathrm{~Hz}$. Similarly, the Fig. $6 \mathrm{~b}$ square waves are close to $0.25 \mathrm{~Hz}$ and the Fig. $6 \mathrm{c}$ square waves are close to $0.75 \mathrm{~Hz}$. While multiple surfaces used the same frequency of input, the inputs were chosen from different rows of the Hadamard matrix and are orthogonal in the time domain.

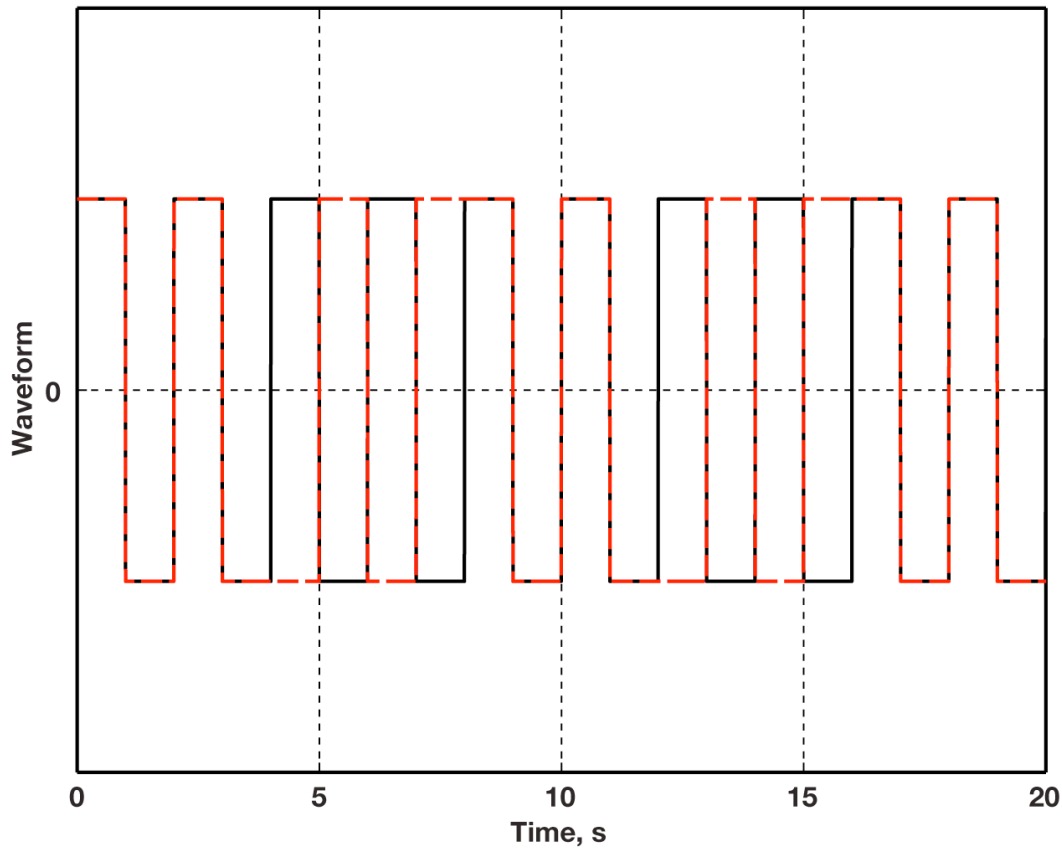

Surface pair 1 --- Surface pair 4

Figure 6a. Input for surface pairs 1 and 4.

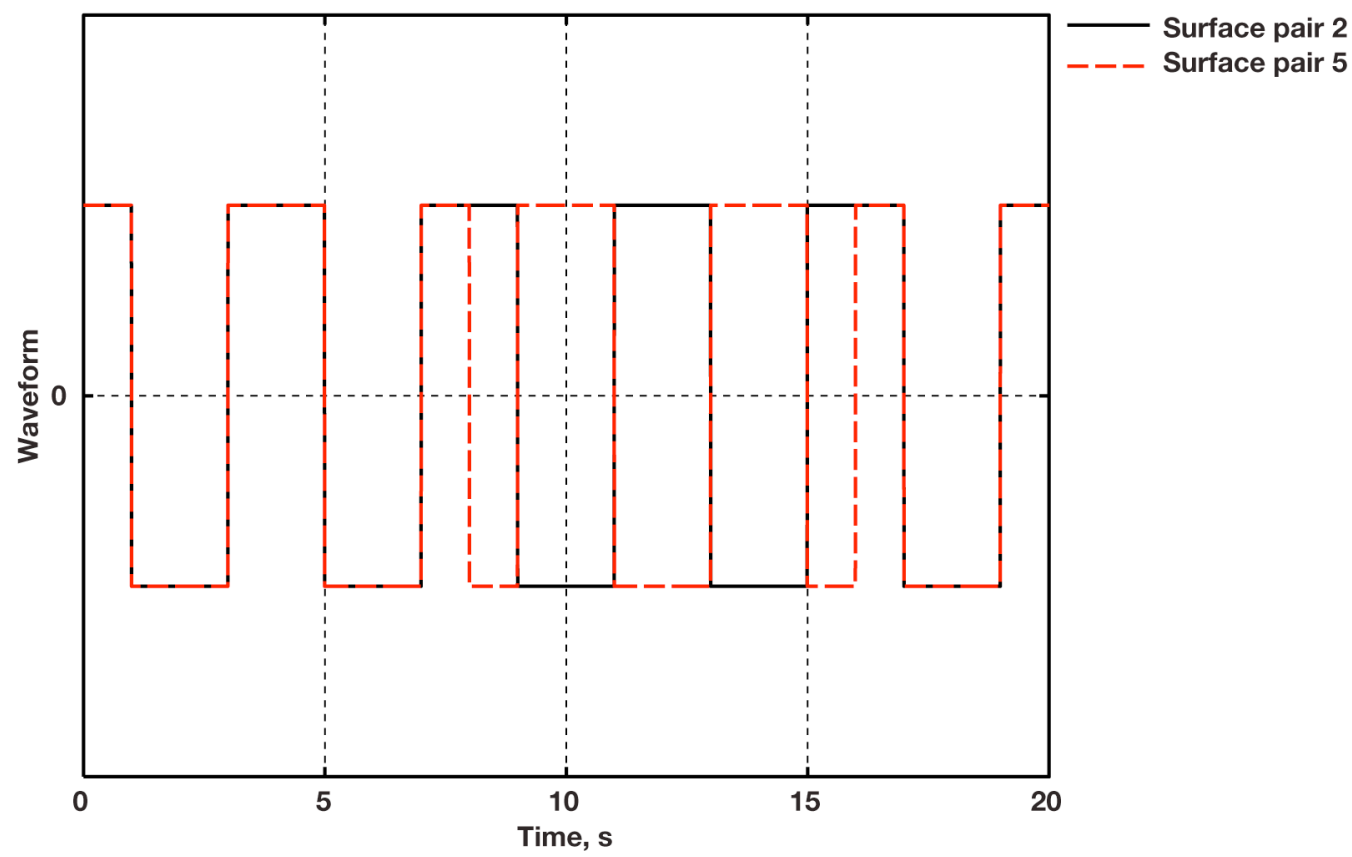

Figure 6b. Input for surface pairs 2 and 5.

American Institute of Aeronautics and Astronautics 


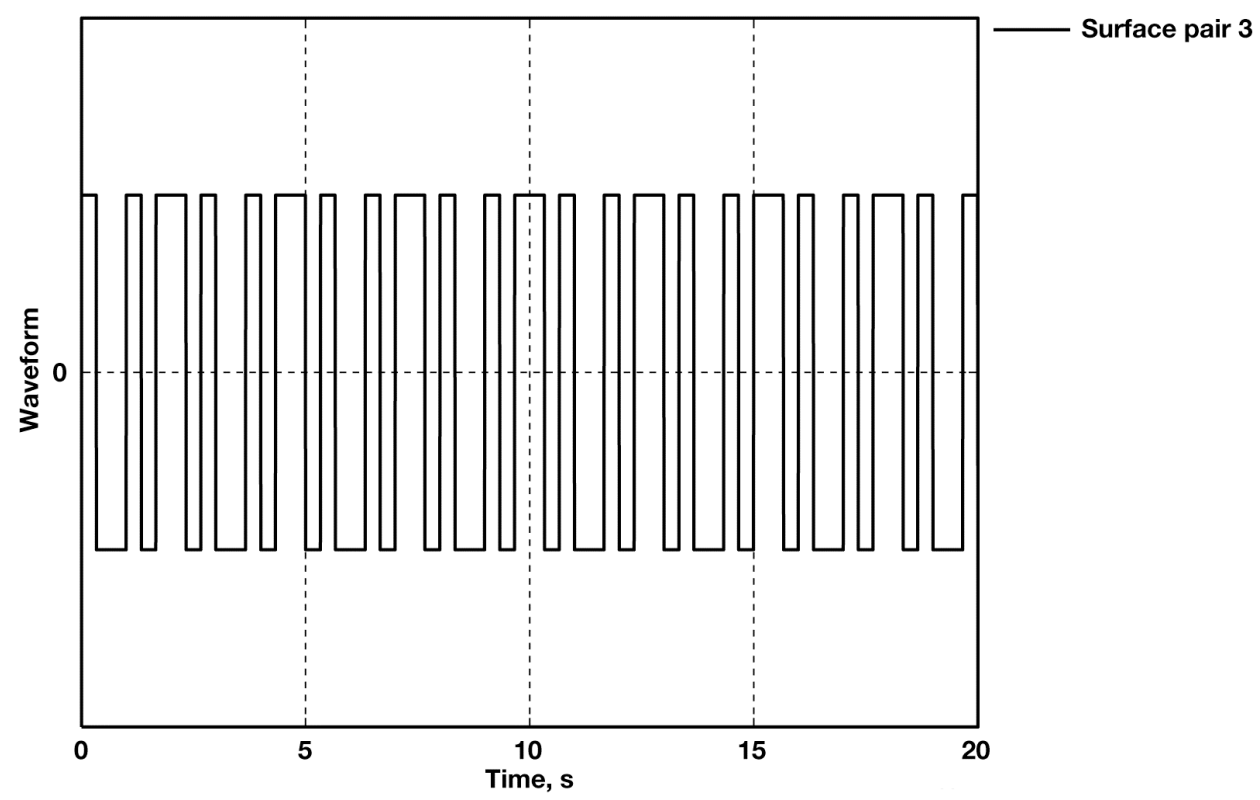

Figure 6c. Input for surface pair 3.

Gains on individual surface pairs were tuned such that all of the surface pairs would elicit a similar magnitude aircraft response. Additionally, there was a single gain that acted on all of the surfaces that could be adjusted by the pilot in flight to account for varying flight conditions.

One complication that was encountered was that many of the excitation inputs could be +1 or -1 simultaneously. This situation would lead to a large aircraft response and move the aircraft away from trim. The use of varying excitation input frequencies on adjacent surfaces helped to alleviate this problem, but did not completely solve it. A limit was imposed on the number of excitation inputs that could simultaneously be the same sign and steps were taken to enforce that limit. First, phase shifting was used to minimize the number of excitations that were the same sign. Next, excitations were zeroed as necessary in order to reduce peak amplitudes of the aircraft response and keep the aircraft near trim during the maneuvers. Figure 7 depicts an example waveform before and after the phase shifting and zeroing process.

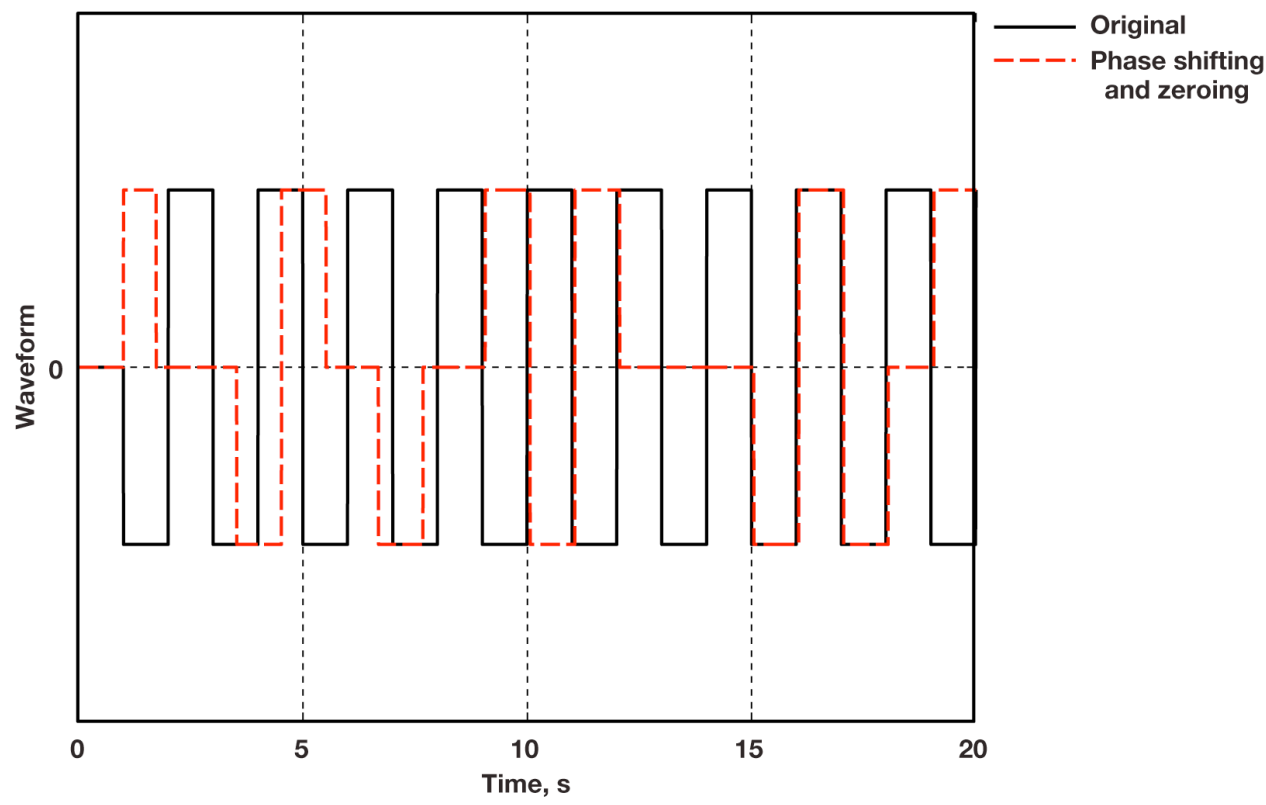

Figure 7. Phase-shifted and zeroed waveform.

American Institute of Aeronautics and Astronautics 
An example of an optimal square wave input both in terms of the base waveform and the measured control surface position is given in Fig. 8.

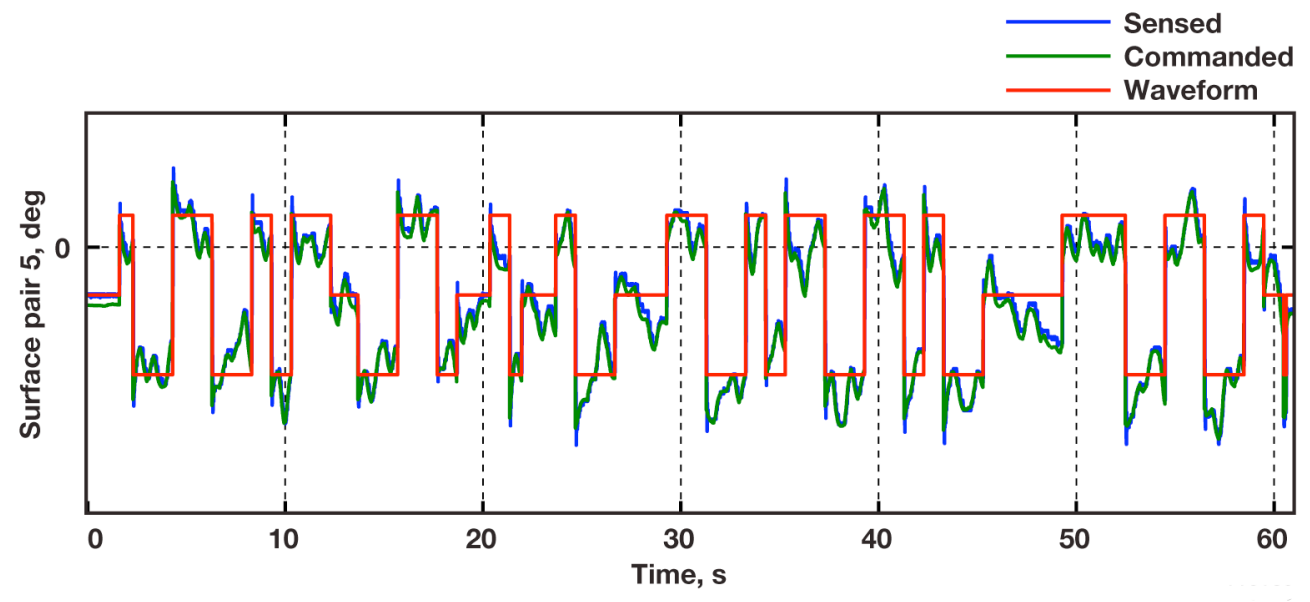

Figure 8. Sample waveform and control surface deflection.

\section{Flight Conditions and Maneuvers}

In order to provide an accurate comparison of the input design methods, the aircraft configuration and flight conditions were chosen to be identical to those used in Ref. 19; that is, a slats-retracted, forward center of gravity configuration between 6 and $11 \mathrm{deg}$ of angle of attack. Each waveform design was flight-tested three times at 6 and 8 deg angle of attack to judge the difference in precision between waveform sets. Waveform set 41 was tested at 10 and 11 deg angle of attack in order to gauge the variation in parameter estimates with angle of attack. Each waveform design was flight-tested for $60 \mathrm{~s}$, although subsets of that flight data were used in analysis in order to draw conclusions on how or if parameter accuracy increases with longer maneuvers.

\section{Flight Results}

Comparing the measured time history response from flight with computed time history response from the parameter estimates can provide a qualitative check of the parameter estimation results. An example for flight data is given in Fig. 9 through Fig. 13. The fit appears good, indicating that all of the system dynamics were captured in the mathematical model used for parameter estimation. 

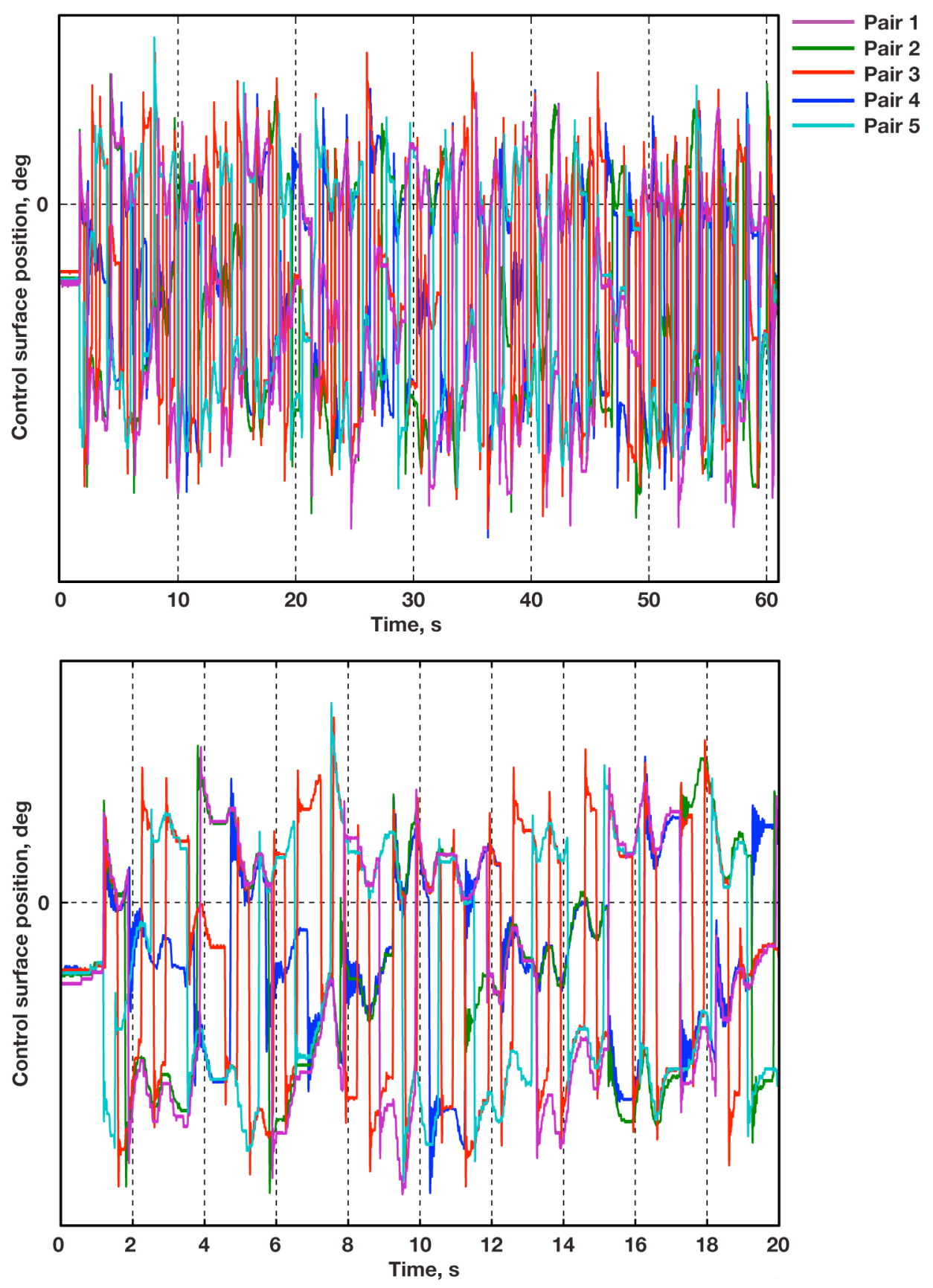

Figure 9. Control surface position. 


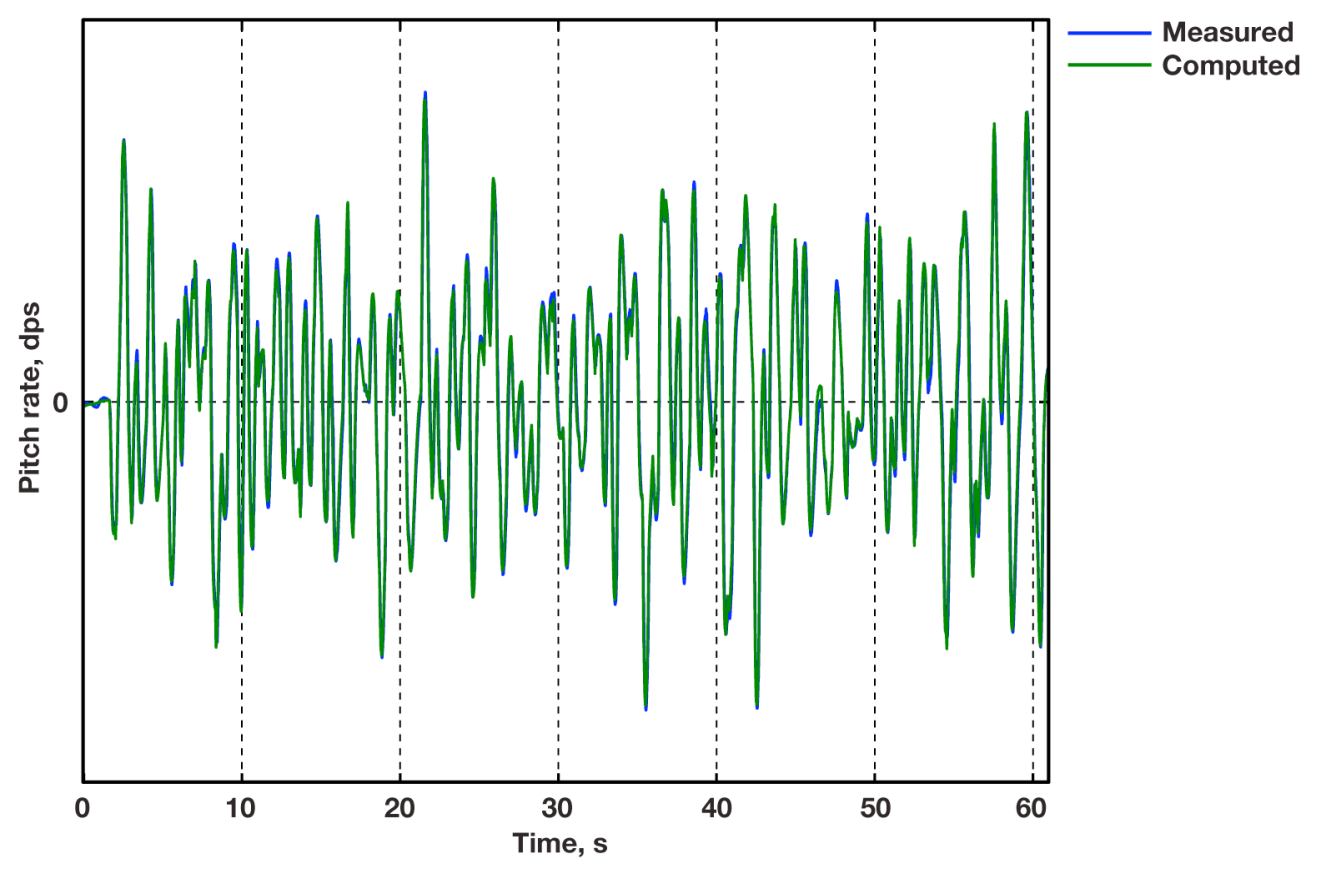

Figure 10. Pitch rate time history.

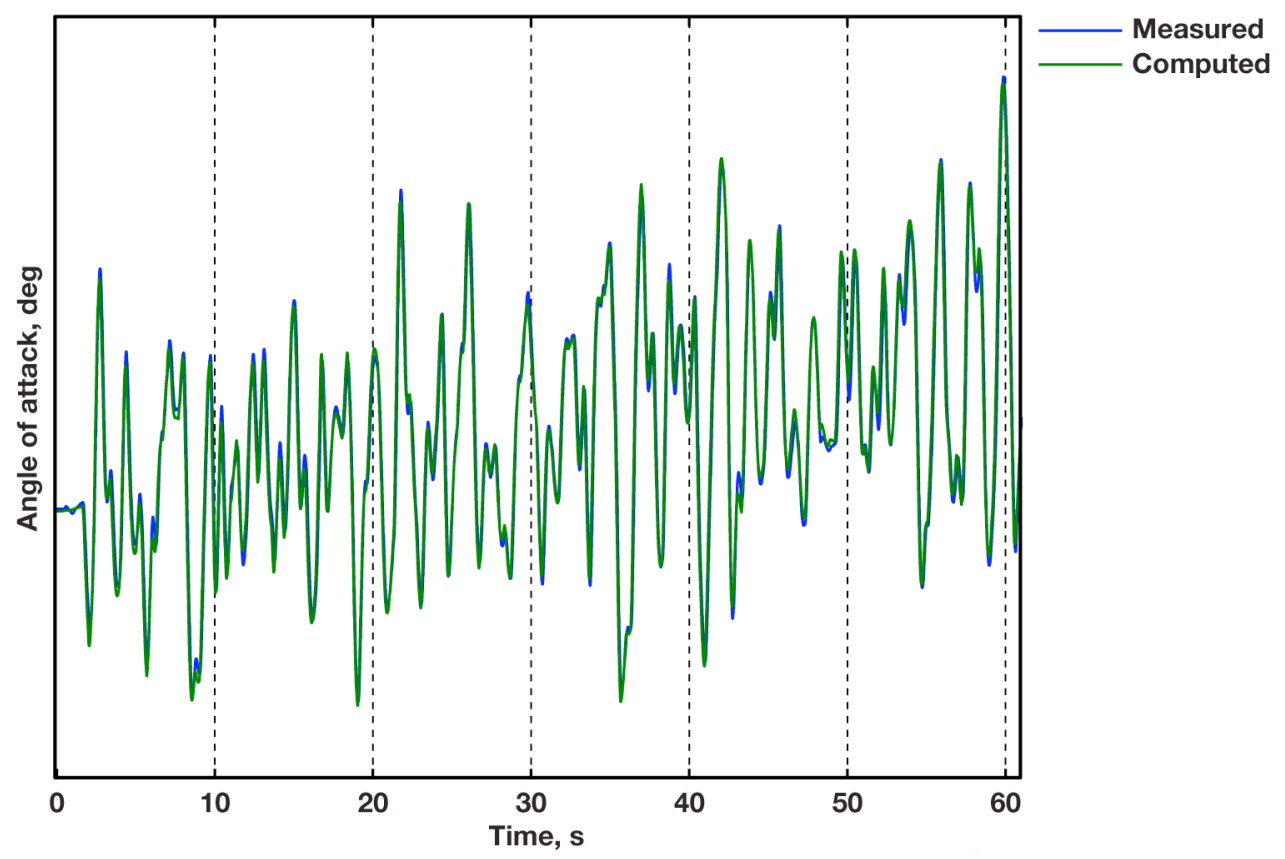

Figure 11. Angle-of-attack time history. 


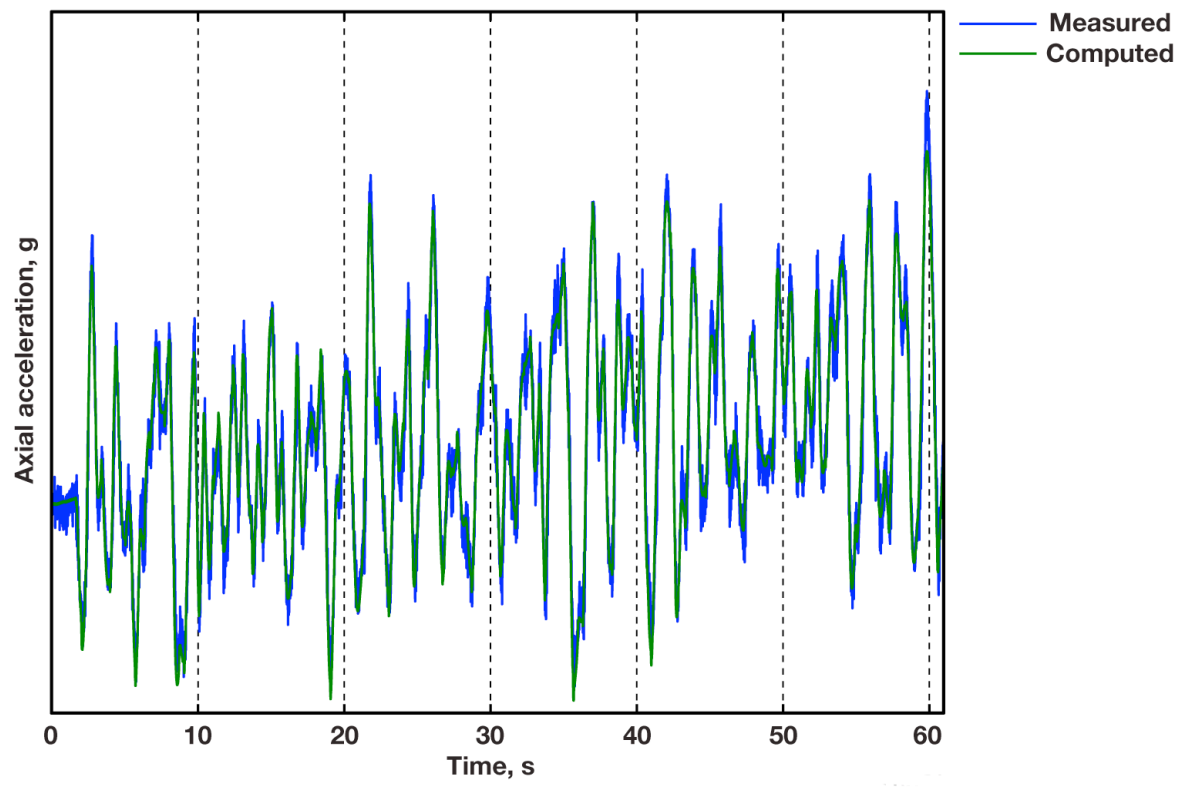

Figure 12. Axial acceleration time history.

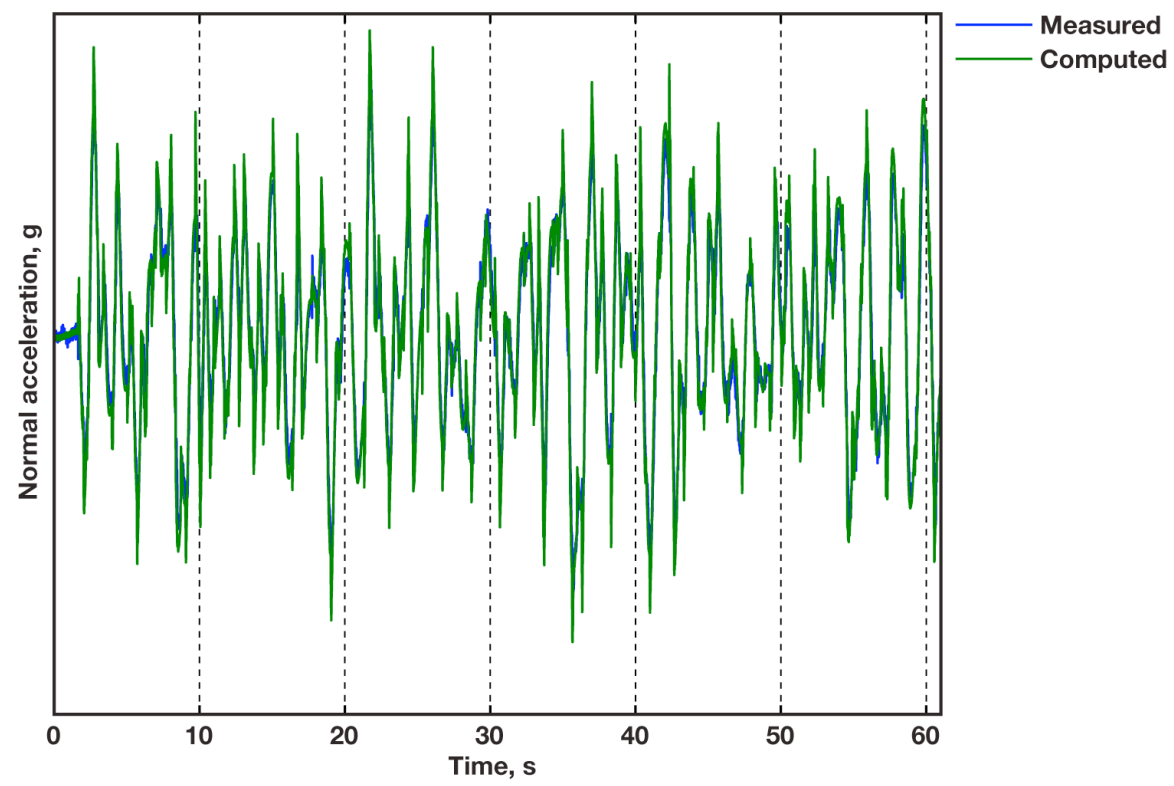

Figure 13. Normal acceleration time history.

Input waveforms were compared with measured surface positions to determine the effect of the closed-loop flight control system with respect to correlation and frequency content. The highest correlation of the input waveform was 10 percent, with most around 5 percent. For flight, the highest correlation was 23 percent, with most around 12 percent. This amount of correlation from the closed-loop flight control system was not considered excessive for parameter estimation. In addition, the frequency content of the input waveforms were not significantly altered by the closed-loop flight control system.

A comparison of flight results using the time separation and orthogonal waveform de-correlation techniques is shown in Fig. 14 for surface pair 5. Error bars on the aerodynamic model are the assumed uncertainty that was used during control law analysis. The metric for judging the input design technique was the precision of the estimates compared with the temporal separation method. In general, the mutually orthogonal square wave inputs produced results with higher precision than temporal separation, especially at 8 and 10 deg angle of attack. This is likely due to the aircraft staying closer to trim during the maneuver. 


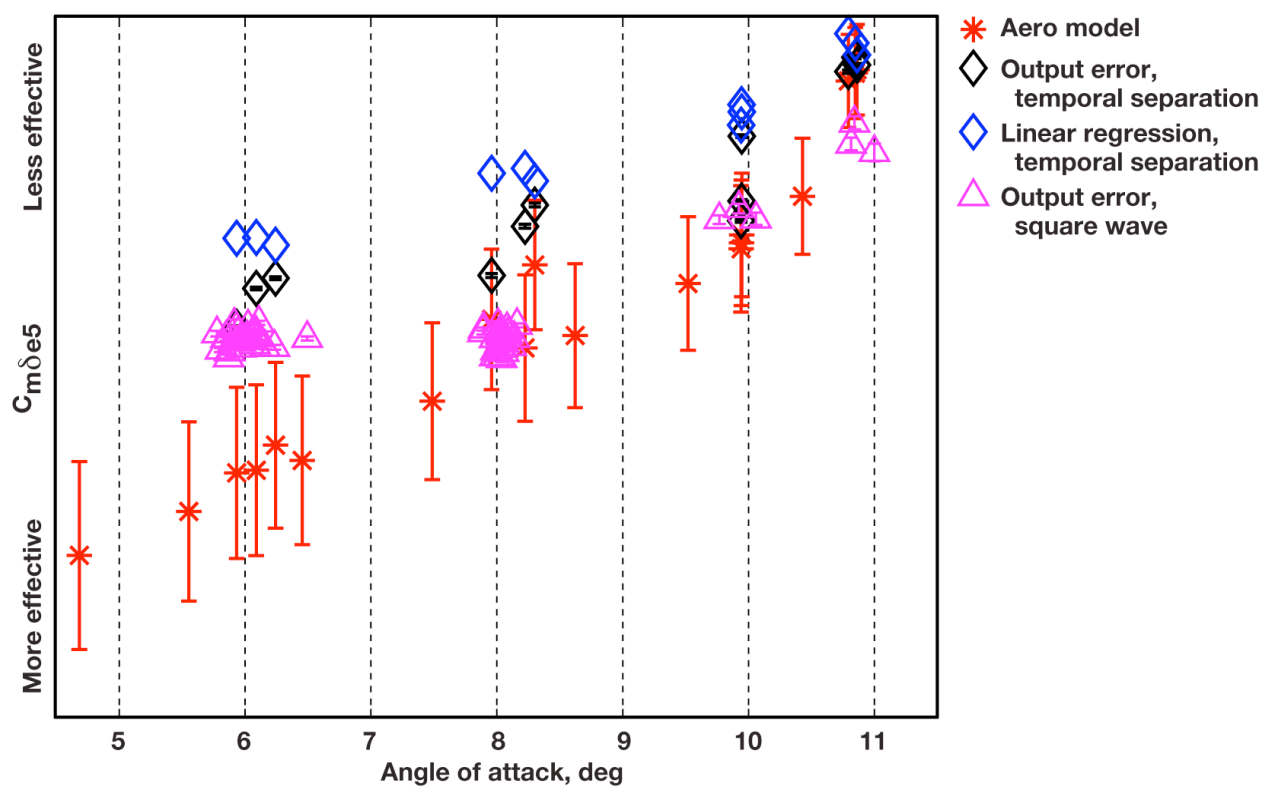

Figure 14. Symmetric surface pair 5: control effectiveness.

It is interesting to notice from Fig. 14 that there appears to be a constant bias between parameter estimates for the temporal separation and square wave input design techniques. Figure 15 shows that the estimates match well if that bias is removed. There was approximately a one-year period of time between the temporal separation flights and the square wave flights during which occurred upgrades to the flight control computer and a full maintenance inspection of the aircraft. Between the two series of flights, the longitudinal moment of inertia changed by approximately 1 percent; however, the same moment of inertia was assumed for parameter estimation of both sets of flight data, which could be a source of the bias in the results. This approach was taken because the original moment of inertia measurements of the aircraft were suspected to be in error, so independent testing was performed after all of the flights were complete. Moment of inertia data from the independent testing was used for all of the parameter estimates.

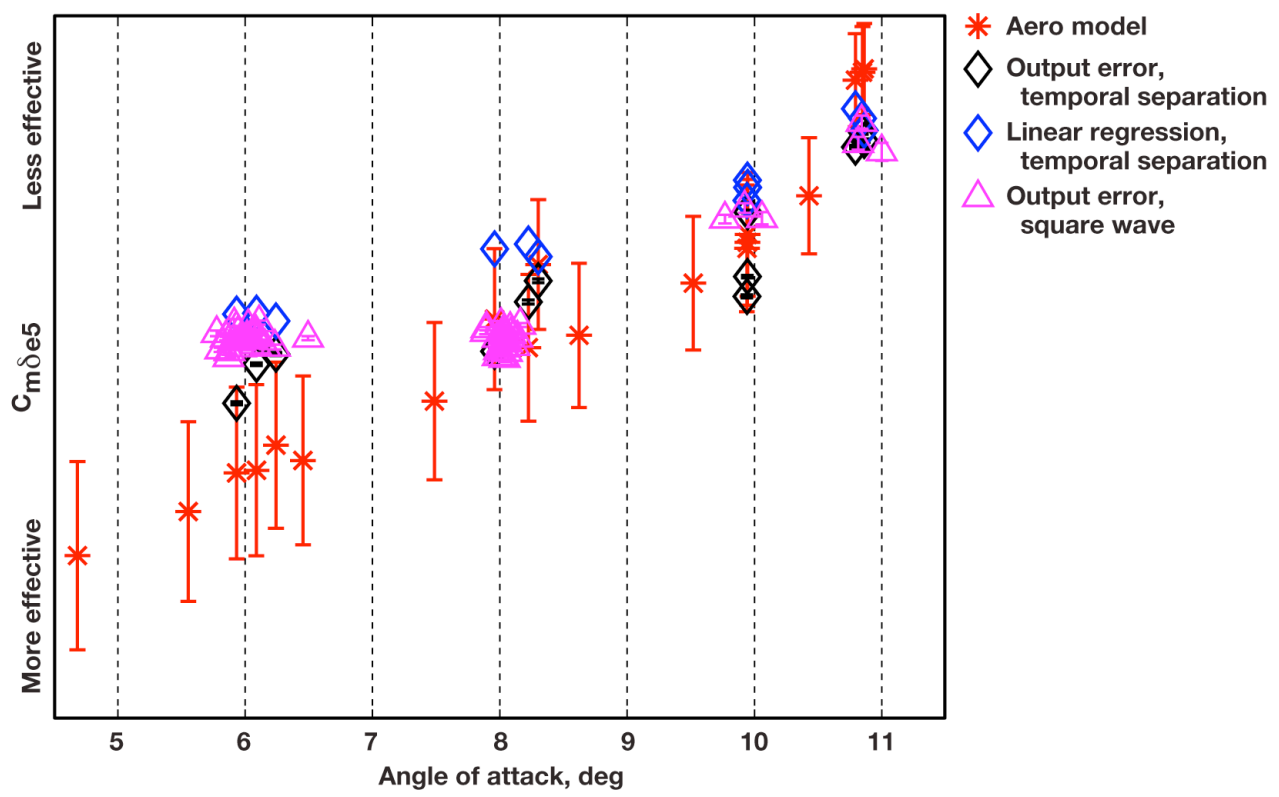

Figure 15. Symmetric surface pair 5: control effectiveness with bias removed. 
A comparison of the waveform sets for control surface pair 5 is shown in Fig. 16 for 6 deg angle of attack. The specific waveform design did not make an appreciable difference in the accuracy or precision of the control surface estimates. The parameter estimation results were not sensitive to the frequency of the input waveform within the range tested: 0.25 to $2.5 \mathrm{~Hz}$.

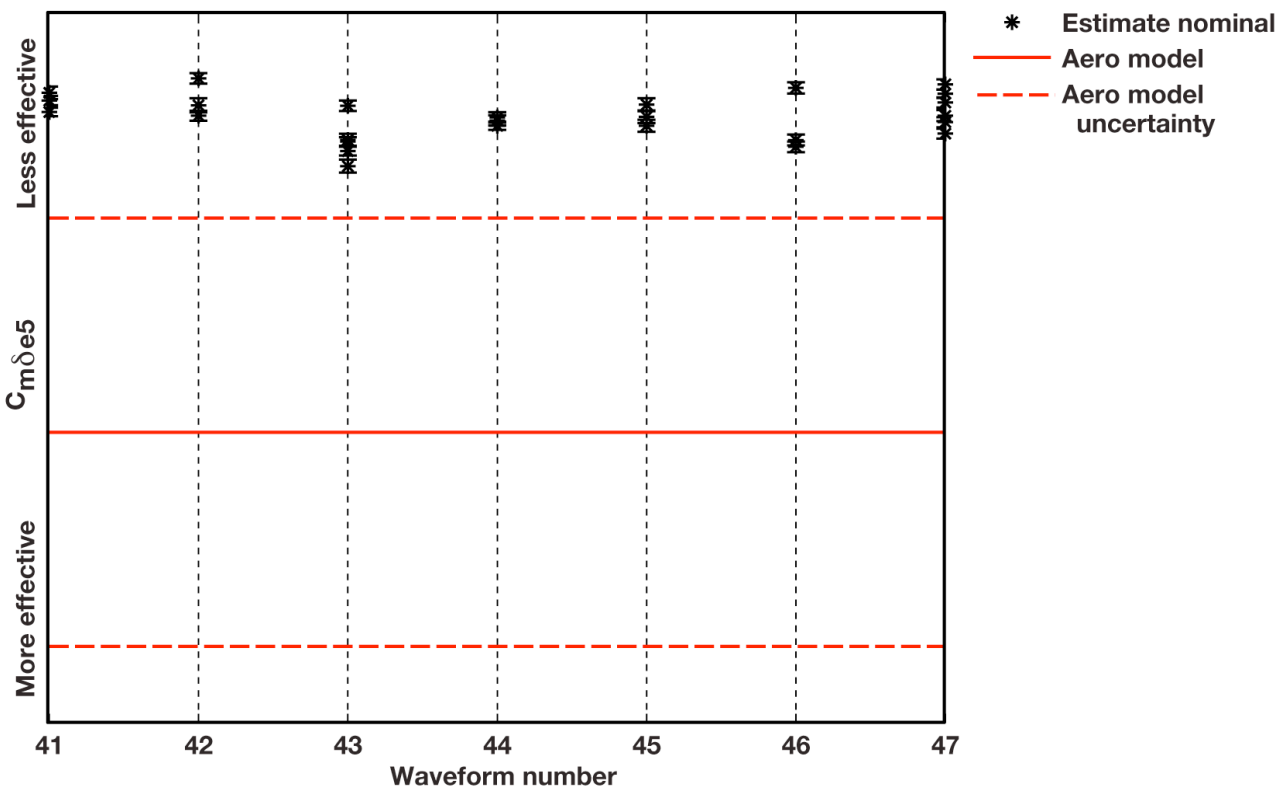

Figure 16. Waveform comparison for 6 deg angle of attack.

Analysis was performed with individual maneuvers to determine how the parameter estimates and Cramér-Rao bounds vary with maneuver length, Fig. 17 for surface pair 5. A 20-s maneuver length appears sufficient to produce accurate parameter estimates. It is interesting to note that longer maneuvers are not necessarily better; it was observed that the Cramér-Rao bounds were worse after $20 \mathrm{~s}$ and improved at approximately $45 \mathrm{~s}$. This is likely because the additional data can add additional uncertainty in the resulting parameter estimate without adding new information in the excitation or response.

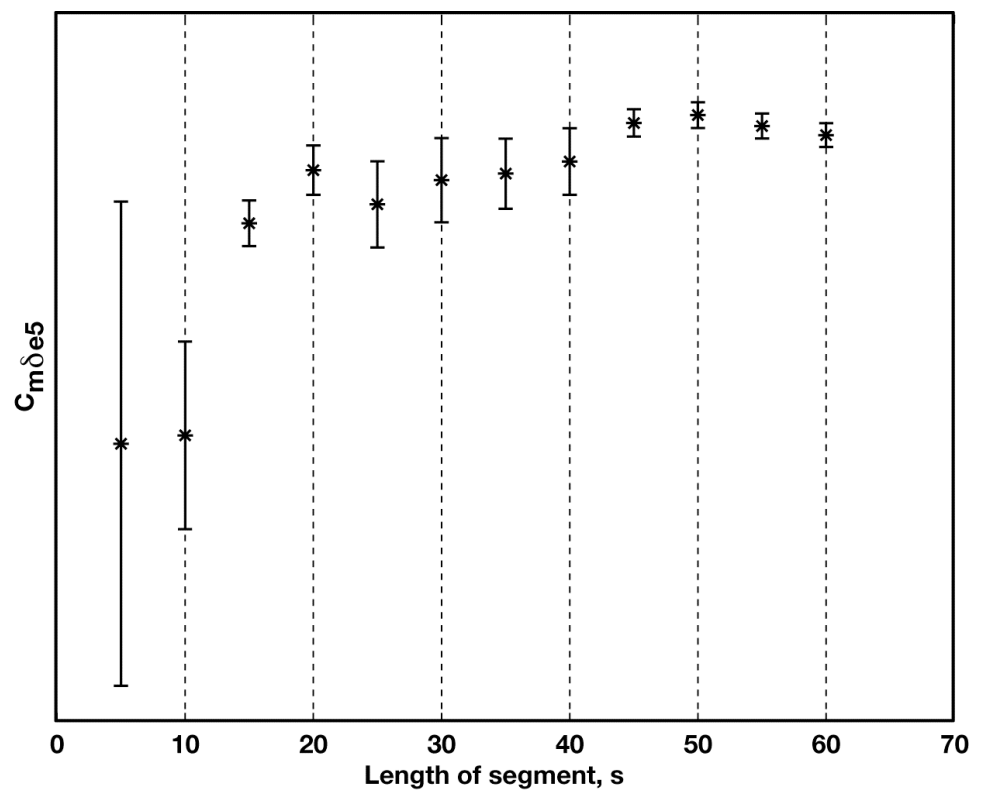

Figure 17. Symmetric surface pair 5: control effectiveness per segment length. 


\section{Conclusion}

A technique for designing parameter estimation inputs for hybrid-wing-body aircraft was presented using X-48B flight data. This technique allows for the estimation of individual control surface effectiveness for closed-loop systems and enables validation of aerodynamic databases, control laws, and simulations. This research will enable the development of hybrid-wing-body and other aircraft with a large number of coplanar control surfaces in a closed-loop control system.

Preliminary analysis of the flight data appears to support the use of mutually orthogonal waveforms as a method for determining individual control effectiveness of aircraft with many actuators and augmentation. Parameter estimates appear to be more precise with the orthogonal waveform method than the temporal separation method. There was no appreciable difference between waveform designs in the estimation results for the frequency range tested. Finally, maneuver lengths of $20 \mathrm{~s}$ should be adequate for most applications of control effectiveness estimation.

\section{References}

${ }^{1}$ Collier, F., "Overview of NASA's Environmentally Responsible Aviation (ERA) Project," 48th AIAA Aerospace Sciences Meeting, Orlando, Florida, January 4, 2010.

${ }^{2}$ Taylor, B. R., and Ratnayake, N. A., "Simulation and Flight Evaluation of a Parameter Estimation Input Design Method for Hybrid-Wing-Body Aircraft," AIAA-2010-7949, 2010.

${ }^{3}$ Smith, M. S., Moes, T. R., and Morelli, E. A., "Real-Time Stability and Control Derivative Extraction From F-15 Flight Data," NASA/TM-2003-212027, 2003.

${ }^{4}$ Morelli, E. A., "Flight Test Experiment Design for Characterizing Stability and Control of Hypersonic Vehicles," AIAA-2008-1682, 2008.

${ }^{5}$ Risch, T. K., "Blended Wing-Body (BWB) Low Speed Vehicle X-48B Blocks 1 and 2 Flight Data Report," NASA/TM-2010-214653/VOL1, 2010, (available from the X-48B project manager, Dryden Flight Research Center, Edwards, California).

${ }^{6}$ Risch, T., Cosentino, G., Regan, C., Kisska, M., and Princen, N., "X-48B Flight Research - Test Progress and Instrumentation Needs for the Future," ITEA 13th Annual Test Instrumentation Workshop, Ridgecrest, California, May 12-14, 2009.

${ }^{7}$ Steers, S. T., and Iliff, K. W., "Effects of Time-Shifted Data on Flight-Determined Stability and Control Derivatives, NASA TN D-7830, 1975.

${ }^{8}$ Iliff, K. W., Maine, R. E., and Montgomery, T. D., "Important Factors in the Maximum Likelihood Analysis of Flight Test Maneuvers," NASA TP-1459, 1979.

${ }^{9}$ Maine, R. E., and Iliff, K. W., "Application of Parameter Estimation to Aircraft Stability and Control, The Output-Error Approach,” NASA RP-1168, 1986.

${ }^{10}$ Maine, R. E., and Iliff, K. W., "Identification of Dynamic Systems - Theory and Formulation," NASA RP-1138, 1985.

${ }^{11}$ Trujillo, B. M., "Determination of Lift and Drag Characteristics of Space Shuttle Orbiter Using Maximum Likelihood Estimation Technique," AIAA-86-2225, 1986.

${ }^{12}$ Maine, R. E., and Iliff, K. W., "The Theory and Practice of Estimating the Accuracy of Dynamic Flight-Determined Coefficients," NASA RP-1077, 1981.

${ }^{13}$ Brasil Neto, N. S., Hemerly, E. M., and Sandoval Goes, L. C., "Aircraft Parameter Estimation Experiment Design Considering Measurement Colored Residuals,” Journal of Aircraft, Vol. 46, No. 6, 2009, pp. 1857-1865.

${ }^{14}$ Morelli, E. A., "F-18 High Alpha Research Vehicle (HARV) Parameter Identification Flight Test Maneuvers for Optimal Input Design Validation and Lateral Control Effectiveness," NASA Contractor Report 198248, 1995.

${ }^{15}$ Morelli, E. A., "Flight Test Validation of Optimal Input Design and Comparison to Conventional Inputs," AIAA-97-3711, 1997.

${ }^{16}$ Morelli, E. A., "Flight-Test Experiment Design for Characterizing Stability and Control of Hypersonic Vehicles," Journal of Guidance, Control, and Dynamics, Vol. 32, No. 3, 2009, pp. 949-959.

${ }^{17}$ Morelli, E. A., "Multiple Input Design for Real-Time Parameter Estimation in the Frequency Domain," 13 th IFAC Symposium on System Identification, 2003, pp. 659-664.

${ }^{18}$ Morelli, E. A., "Practical Input Optimization for Aircraft Parameter Estimation Experiments," NASA CR-191462, 1993.

${ }^{19}$ Morelli, E. A., and Klein, V., "Optimal Input Design for Aircraft Parameter Estimation Using Dynamic Programming Principles,” AIAA-90-2801-CP, 1990. 
Flight Test of Orthogonal Square Wave Inputs for Hybrid-Wing-Body Parameter Estimation

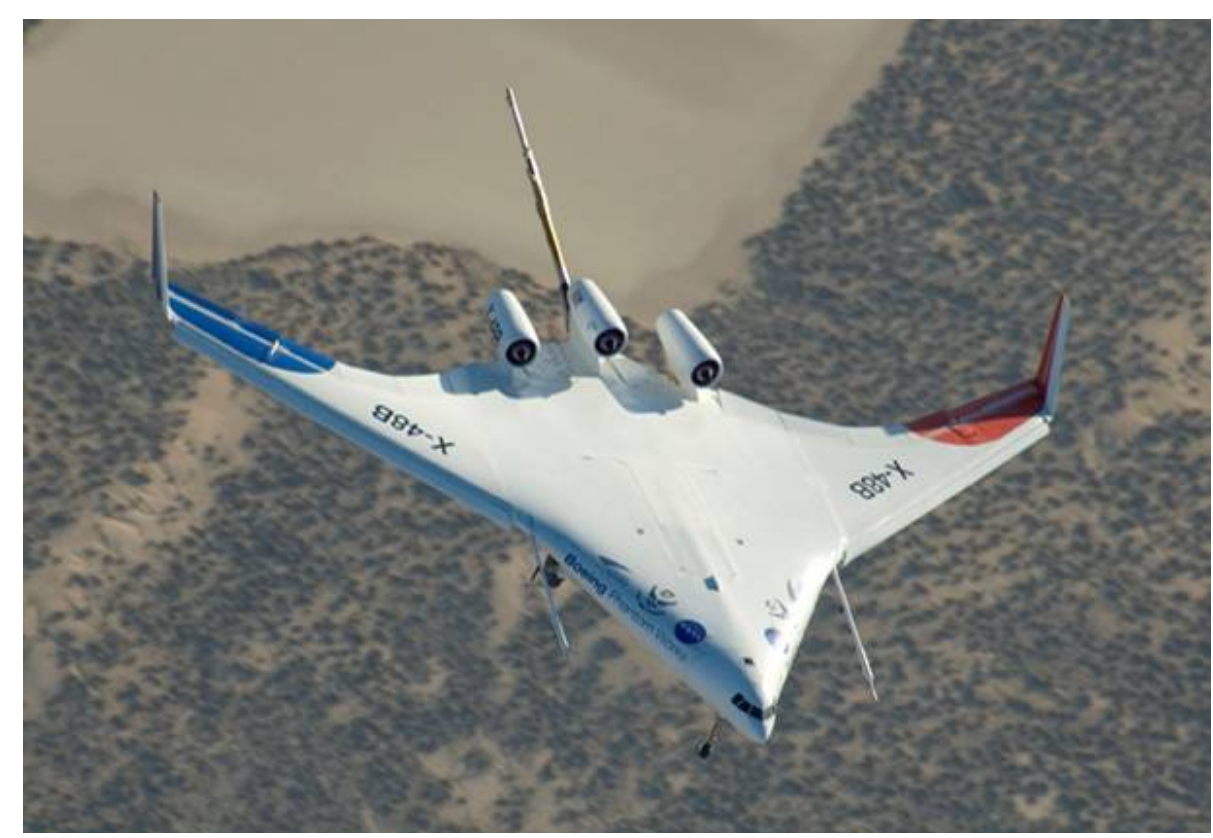

NASA Dryden Flight Research Center

\author{
Brian R. Taylor
}

Nalin A. Ratnayake 


\section{Agenda}

- Motivation

- Aircraft Background

- Correlation

- Input Design Methods for De-correlation

- Approach

- Waveform Design

- Flight Conditions and Maneuvers

- Results

- Parameter Estimates

- Waveform Comparison

- Maneuver Length

- Conclusions 


\section{Motivation}

- Environmentally Responsible Aviation project goal:

- Improve noise and efficiency of future aircraft

- Hybrid-Wing-Body (HWB) aircraft have potential to reduce fuel burn and noise compared with current aircraft

- Parameter estimation used to validate aerodynamic models, simulations, and control law analysis

- Correlation challenges

- Many nearly coplanar, multi-objective control surfaces

- Closed-loop flight control system

$>$ Estimate single surface control effectiveness with appropriate input design methods 


\section{X-48B Background}

- Research partnership of Boeing, NASA, and AFRL

- Design and fabrication contracted to Cranfield Aerospace

- Airframe

- Remotely piloted from ground control station

- $8.5 \%$ dynamically scaled (rigid body)

- Wingspan: $20.4 \mathrm{ft}$

- Weight: $525 \mathrm{lbf}$

- Thrust: $54 \mathrm{lbf}$ each (3 JetCat turbojet engines)

- Closed-loop flight control system

- 20 control surfaces

- 4 split ailerons

- 2 winglet rudders

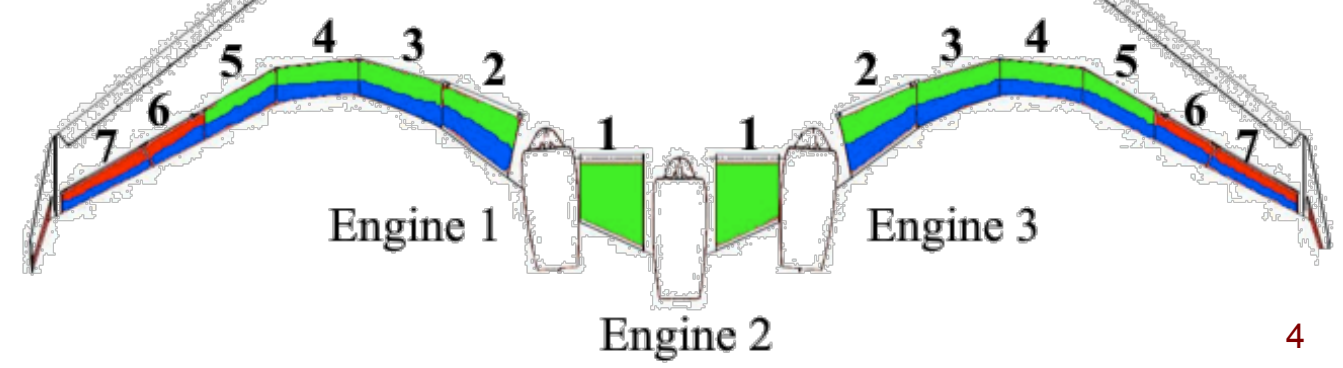




\section{Correlation}

- Necessary to identify the response attributable to each input

- Redundant, multi-objective, nearly co-planar control surfaces evoke similar aircraft responses

- Closed-loop flight control system treats excitations as disturbances and attempts to damp them out with the use of the remaining control surfaces

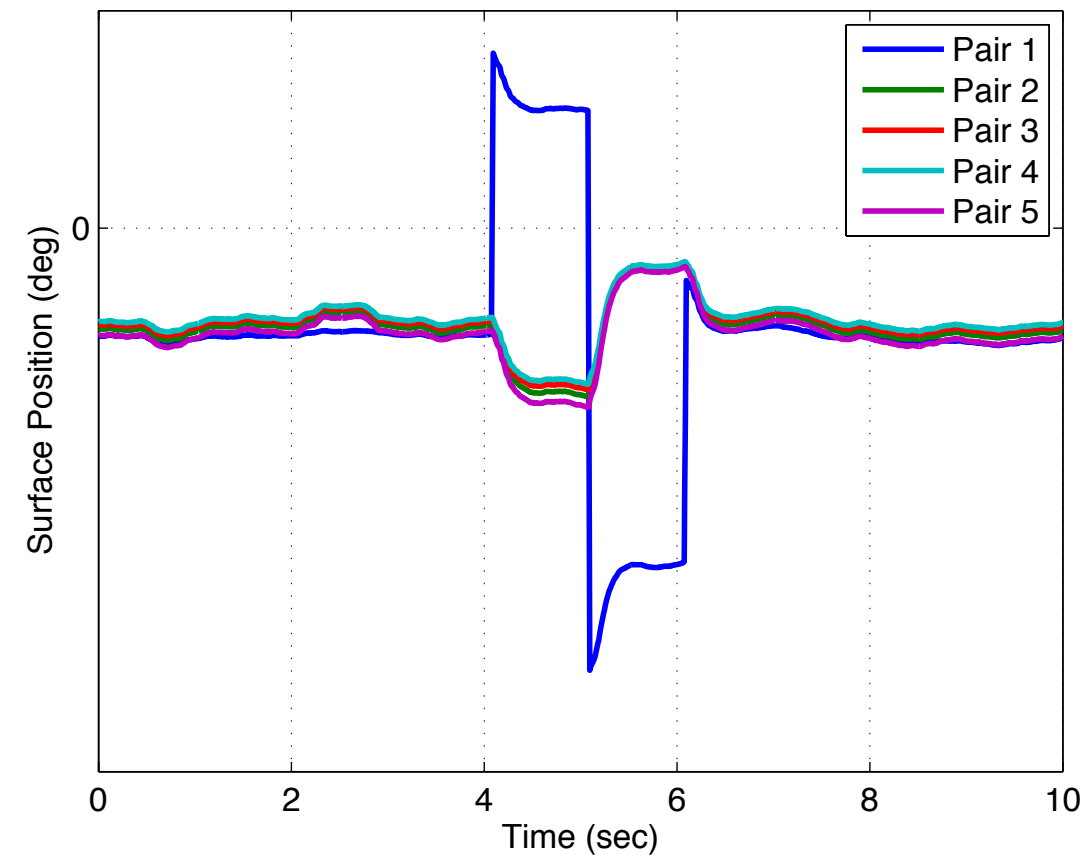




\section{Input Design Methods for De-correlation}

- Aerodynamic model

- Only the parameters corresponding to the excited input are estimated

- Errors in the aerodynamic model can propagate to the estimate

- Temporal separation

- Excite each surface capable of contributing to the aircraft response individually by a doublet and separate by time

- Requires a significant amount of flight time

- Potential inaccuracies caused by differing initial conditions

- Multi-sines

- Excites each surface capable of contributing to the aircraft response simultaneously with sine-waves which are mutually orthogonal in the frequency domain

- Limited aircraft response amplitude to stay near trim

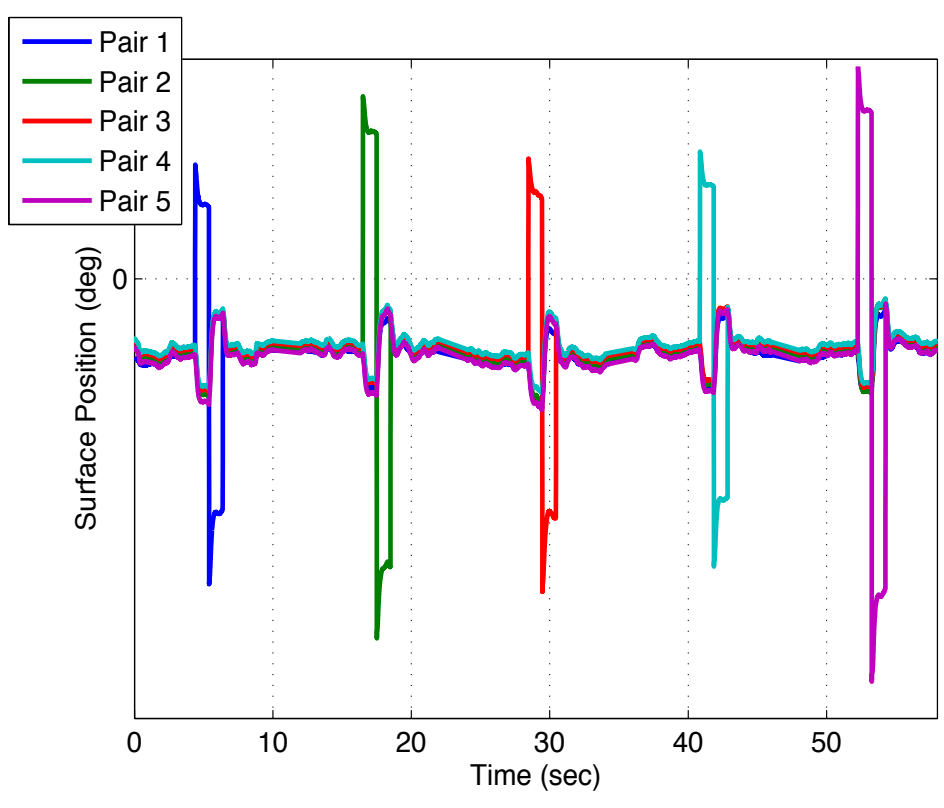




\section{Approach}

- Square waves which are mutually orthogonal in the time domain

- Further de-correlation by using different "frequencies" for adjacent surfaces

- Planned use of time domain output-error parameter estimation tools

- Limited amplitude to keep the aircraft near trim

- Chose input frequency near $1 \mathrm{~Hz}$

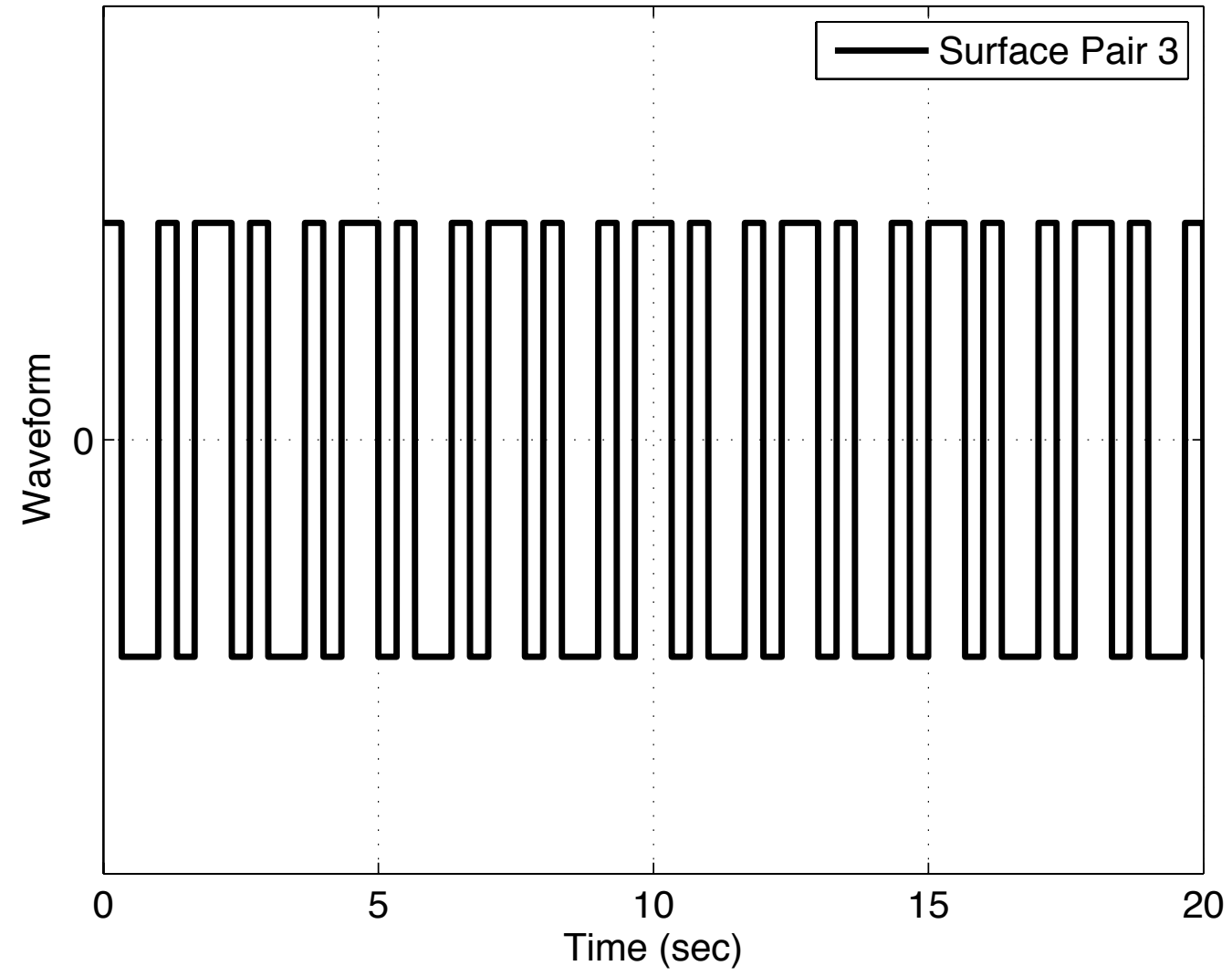




\section{Waveform Design}

- Hadamard matrix

- Square matrix, entries either +1 or -1

- Mutually orthogonal rows

- Used to create a $1024 \times 1024$ matrix

- Sampled at $200 \mathrm{~Hz}$ to create a 5.12 second maneuver input

- Determined the average frequency of each row after sampling

- Chose inputs rows based on average frequency

- Repeated maneuver to make 60 seconds long

- Even though same frequency, still mutually orthogonal because of different rows
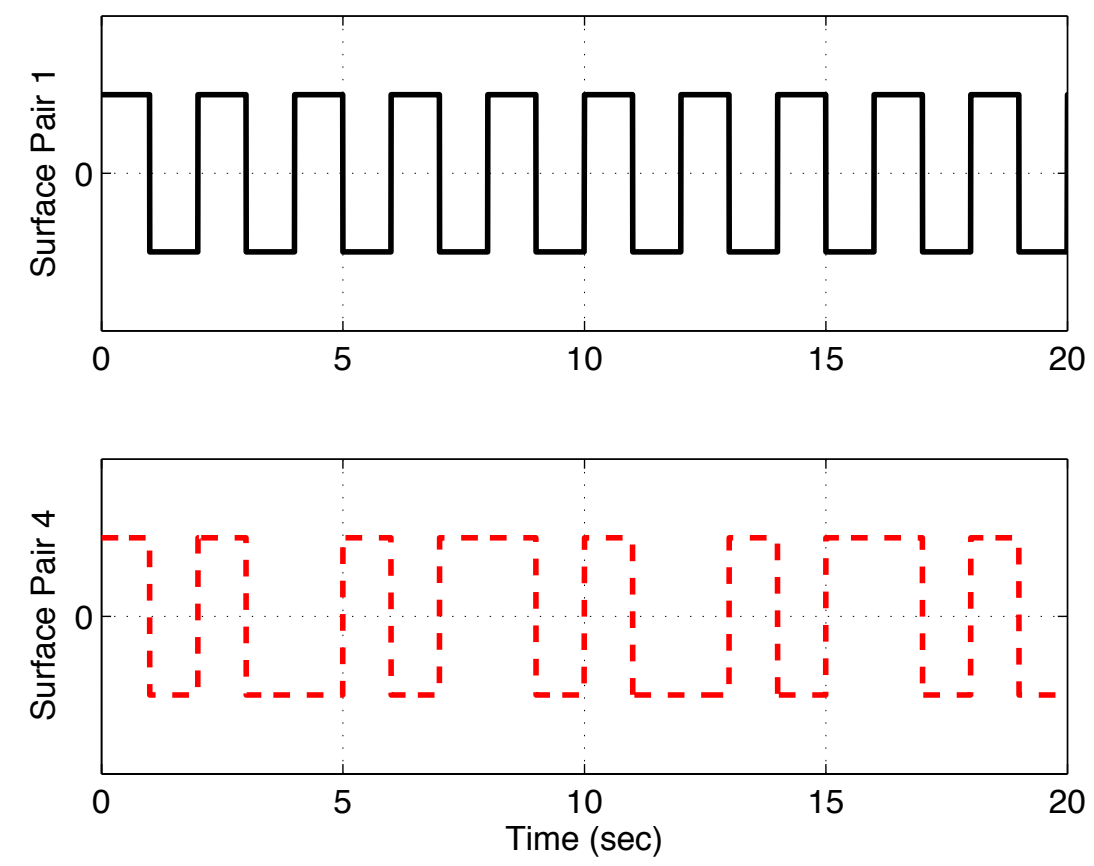


\section{Waveform Design}

- Waveform numbers to define sets of individual surface pair inputs

- 7 waveform sets tested to determine affect of frequency on parameter estimation

- Waveform sets 41 through 44 increasing frequency with $0.25 \mathrm{~Hz}$ separation in frequency for adjacent surfaces

- Waveform sets 45 through 47 increasing frequency with $0.5 \mathrm{~Hz}$ separation in frequency for adjacent surfaces

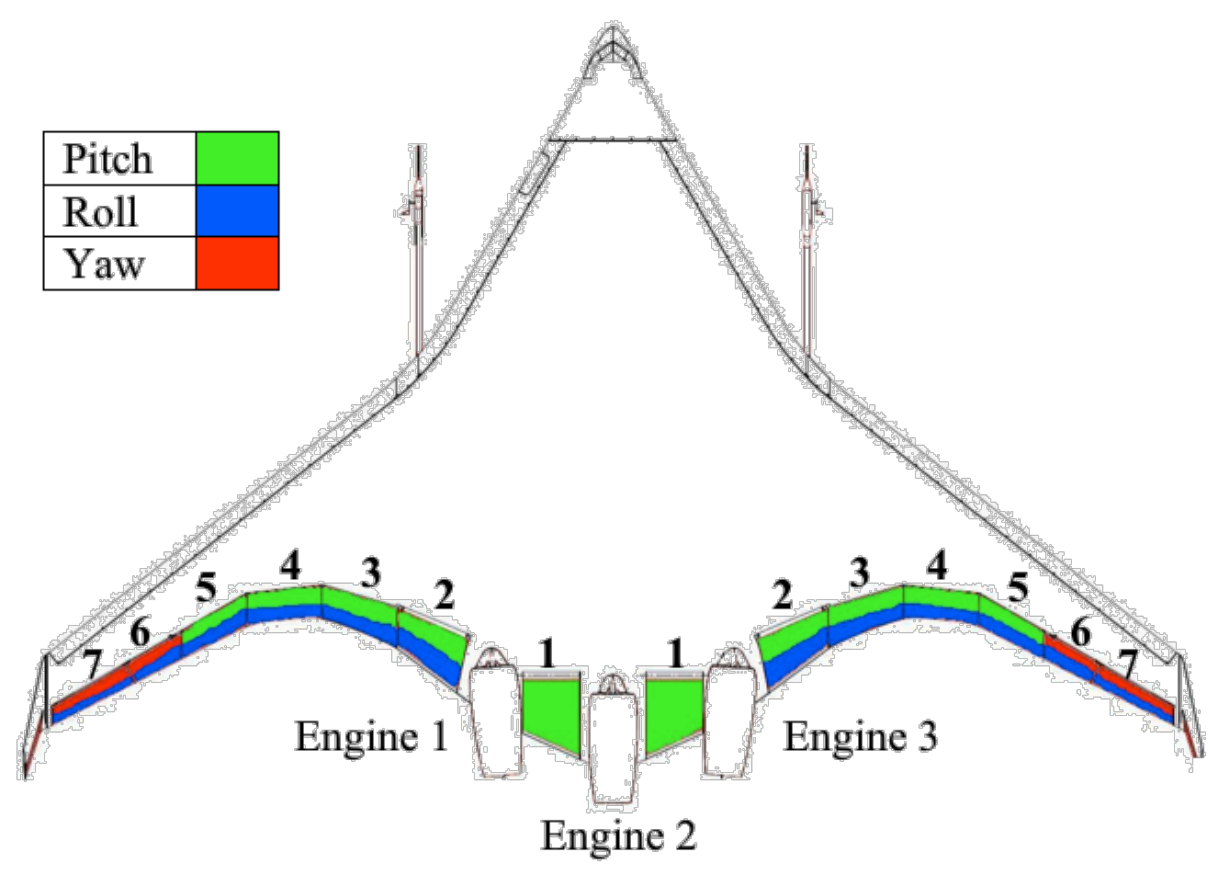

\begin{tabular}{|l|l|l|l|}
\hline \multicolumn{4}{|c}{ Frequency $(\mathrm{Hz})$} \\
\hline Waveform & Pairs 1, 4 & Pairs 2, 5 & Pair 3 \\
\hline 41 & 0.5 & 0.25 & 0.75 \\
\hline 42 & 1.0 & 0.75 & 1.25 \\
\hline 42 & 1.5 & 1.25 & 1.75 \\
\hline 44 & 2.0 & 1.75 & 2.25 \\
\hline 45 & 1.0 & 0.5 & 1.5 \\
\hline 46 & 1.5 & 1.0 & 2.0 \\
\hline 47 & 1.5 & 0.5 & 2.5 \\
\hline
\end{tabular}




\section{Waveform Design}

- Zeroing and phase shifting used to limit the magnitude of the aircraft response

- Maximum of 4 surface pairs simultaneously +1 or -1

- Phase shifting used first, then zeroing during portions of the individual surface pair excitation

- Zeroing spread across all of the surface pairs
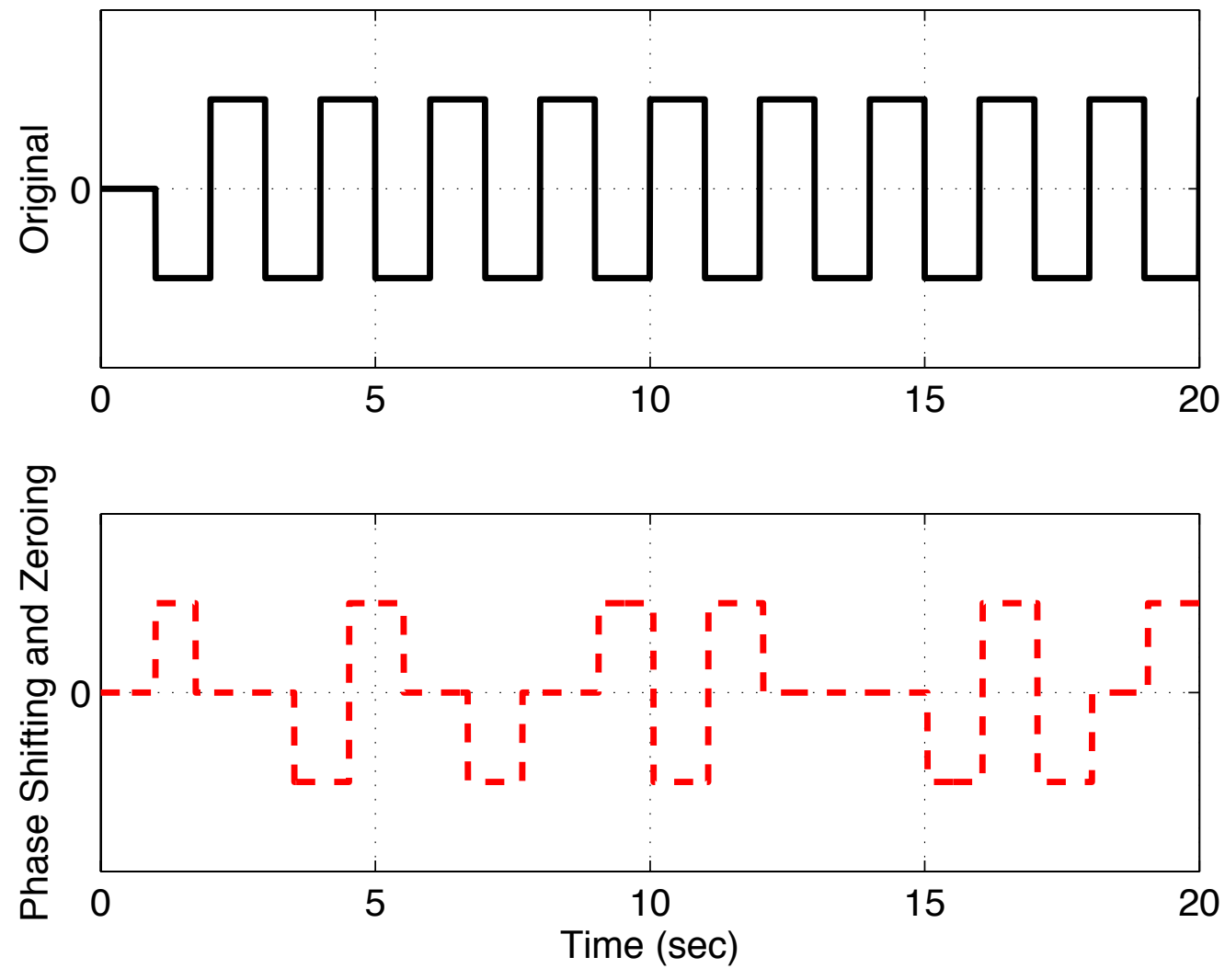


\section{Waveform Design}

- Gains on individual surface pairs tuned such that all of the surface pairs would elicit a similar aircraft response

- Performed so that each individual surface pair would create enough aircraft response for parameter estimation without breaking safety of flight limits

- Keeps the aircraft near trim

- Single gain that was tunable in flight to adjust for flight condition

- Angle of attack variation of $+/-1$ degree angle of attack desired

- Gains tuned in non-linear simulation 


\section{Flight Conditions and Maneuvers}

- Each waveform tested at 6 and 8 degrees angle of attack

- Waveform set 41 tested at 10 and 11 degrees angle of attack

- Three repeats of each maneuver at each flight condition

- Maneuver length of 60 seconds

- Segments of each maneuver used in post-processing to determine the variation in the parameter estimates with respect to maneuver length

- Slats-retracted, forward center of gravity aircraft configuration 


\section{Time History Results}

- Qualitative check of the parameter estimation results

- Good fit; all of the system dynamics were captured in the mathematical model used for parameter estimation

- Investigated correlation caused by the closed-loop flight control system

- Highest correlation of input waveform $10 \%$ with most around $5 \%$

- Highest correlation of flight data $23 \%$ with most around $12 \%$
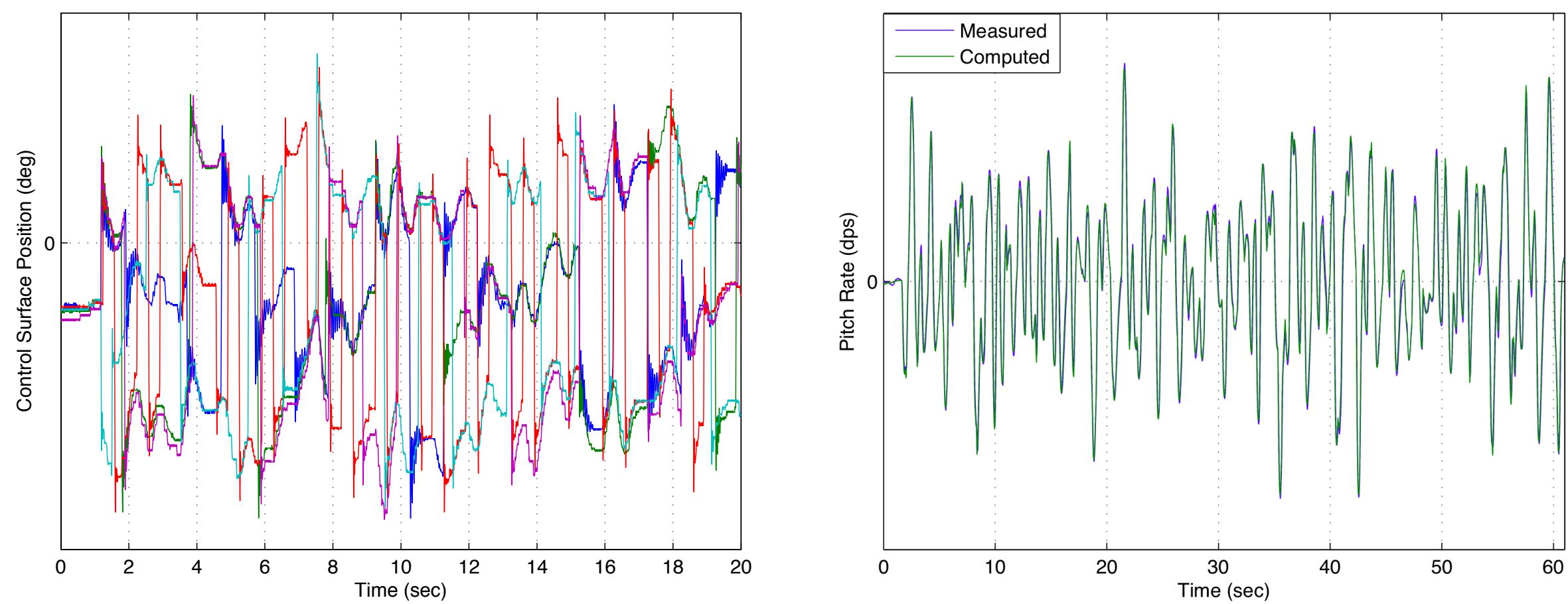


\section{Flight Results}

- Error bars on the aero model are the assumed uncertainty during control law analysis

- Judged based on precision compared to temporal separation

- Results with higher precision likely because of the aircraft staying closer to trim during the maneuver

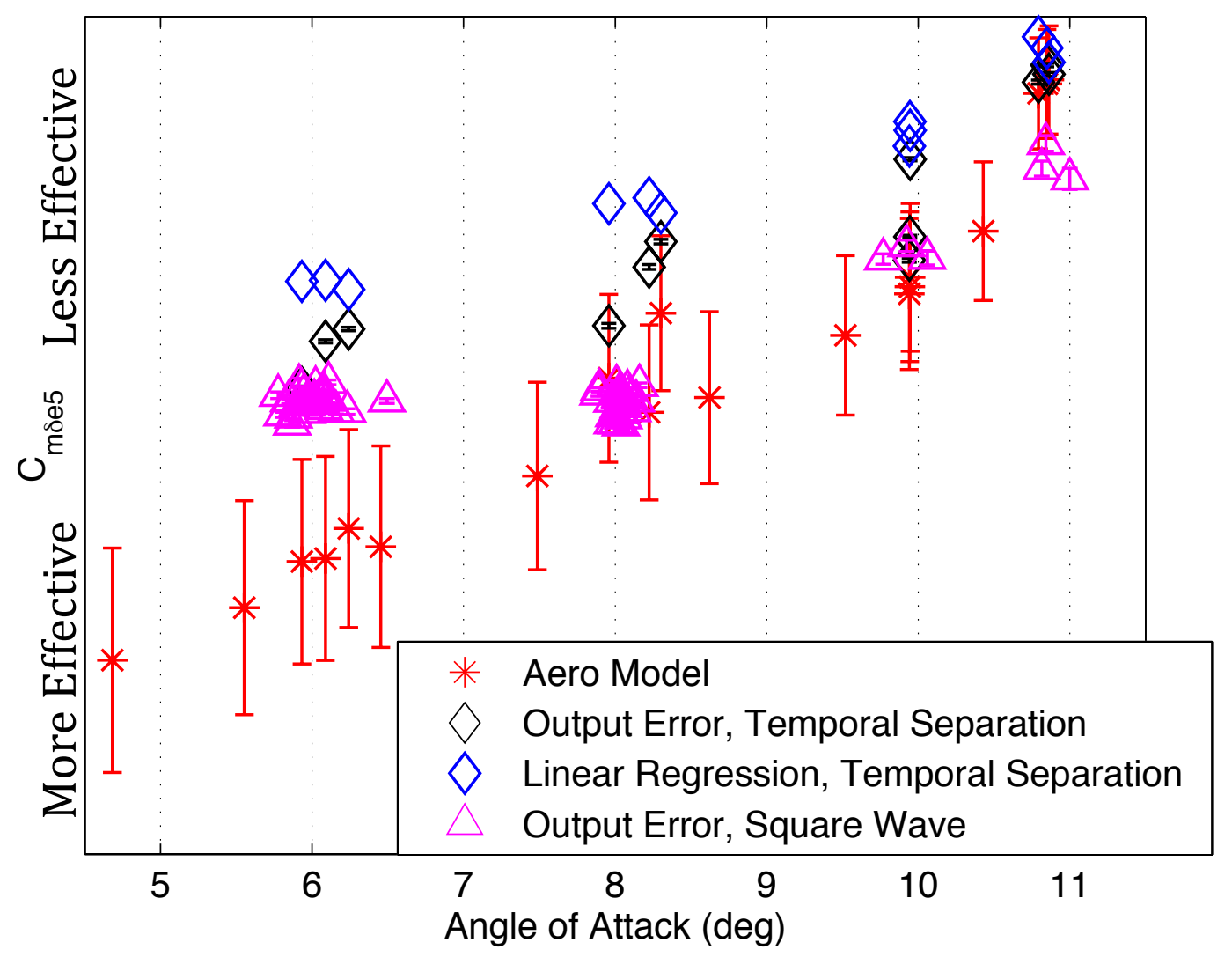




\section{MOI Correction}

- Moment of inertia data from independent testing used for all parameter estimates

- One year of time between temporal separation and square wave flight tests including a full maintenance inspection of aircraft and replacing the flight computer

- $1 \%$ change in longitudinal moment of inertia

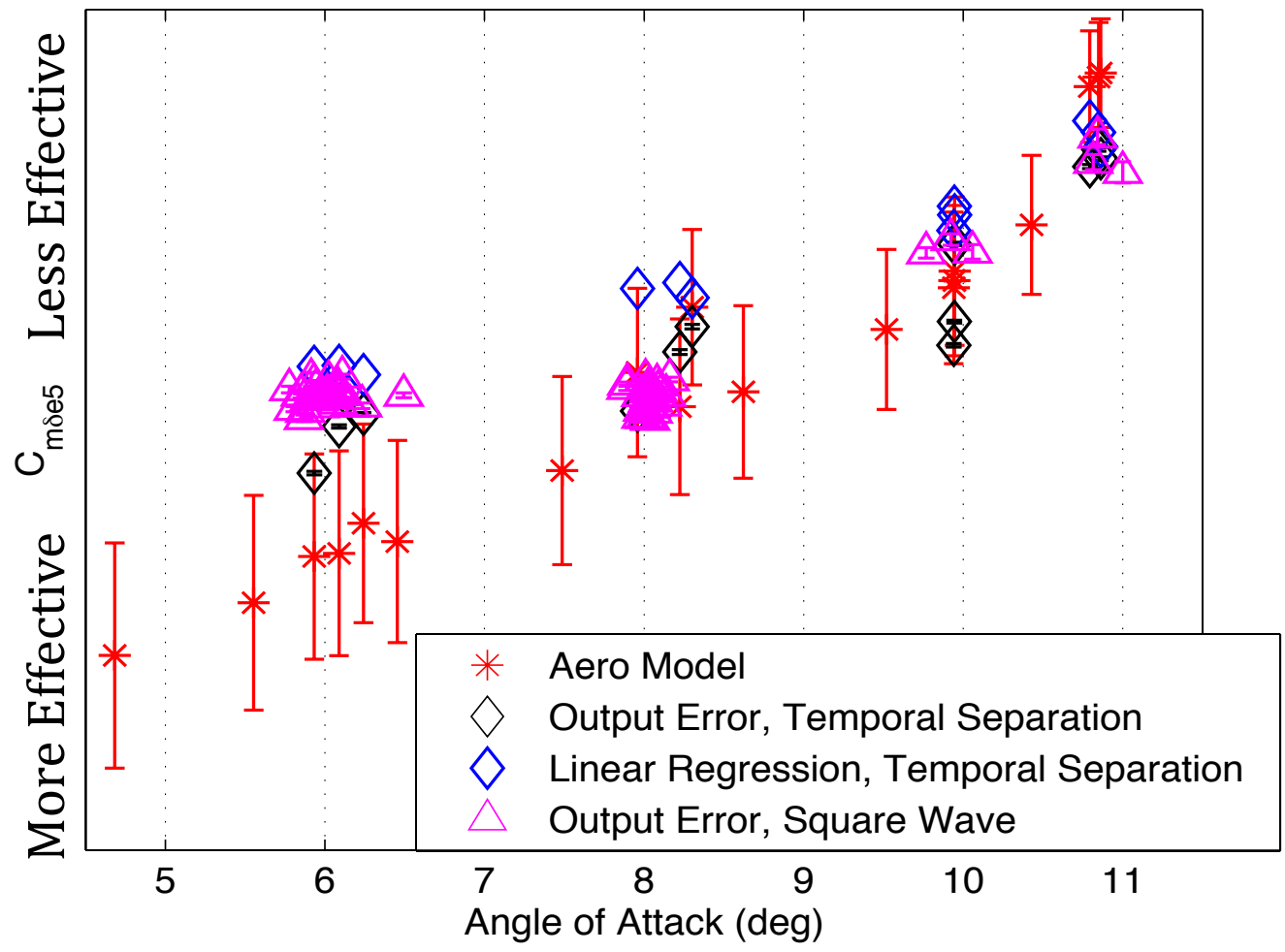




\section{Waveform Comparison}

- 6 degrees angle of attack

- No appreciable difference in accuracy or precision of the control surface estimates

- Not sensitive to the frequency of the input within the range tested, 0.25 to $2.5 \mathrm{~Hz}$

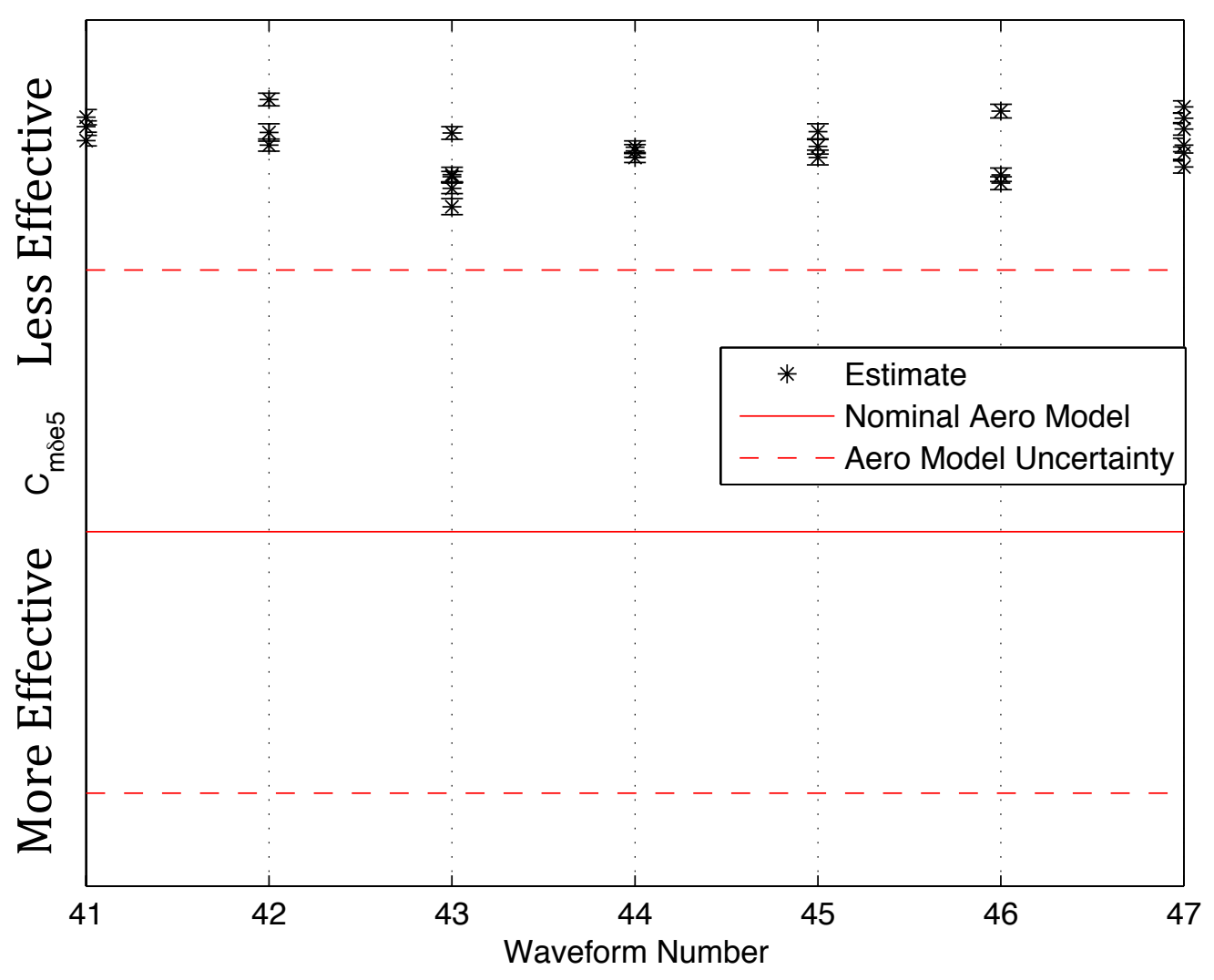




\section{Maneuver Length}

- Analysis with varying length of segments to determine how parameter estimates vary with maneuver length

- Worse after 20 seconds and improve around 45 seconds

- 20 seconds adequate for most applications

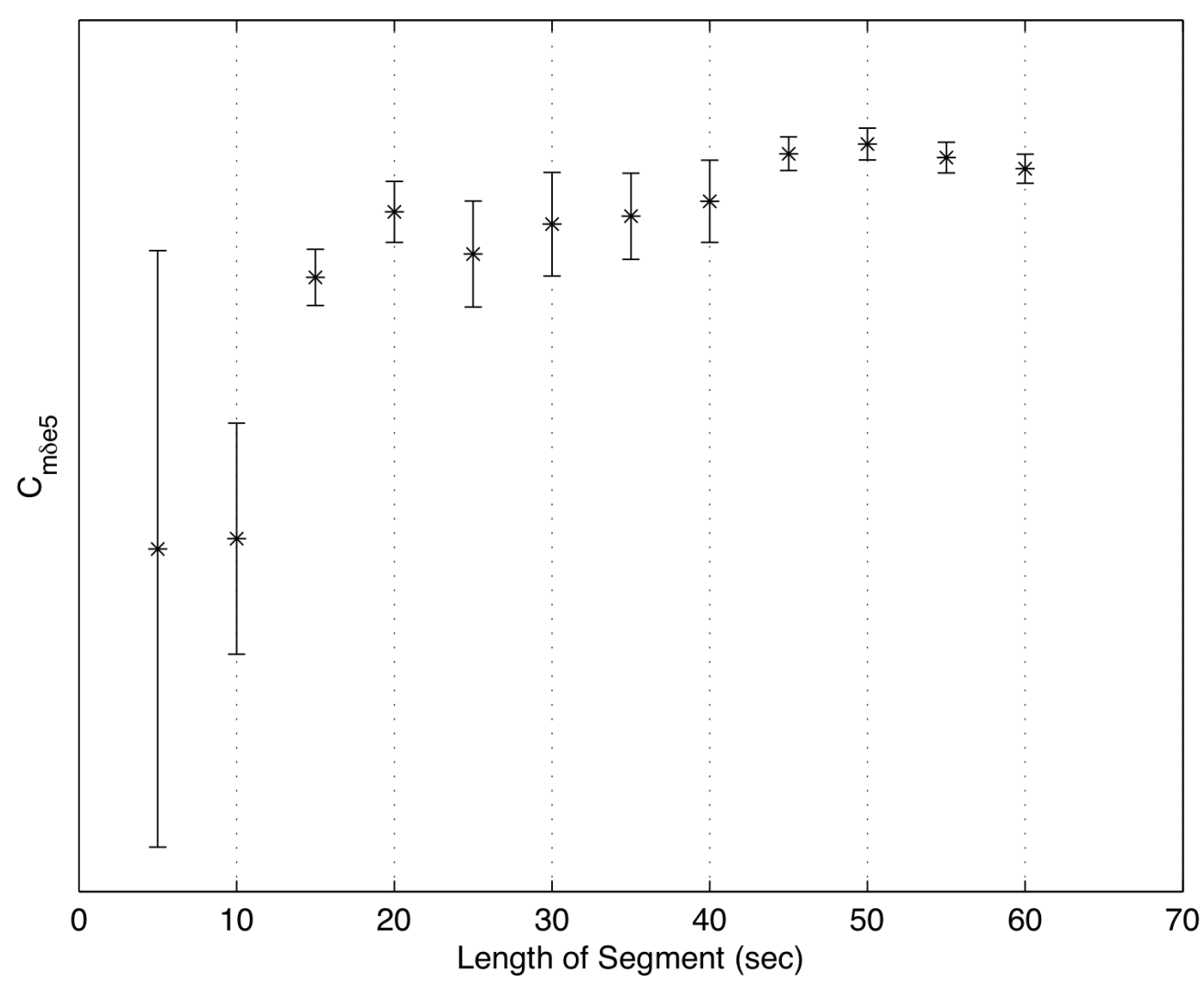




\section{Conclusions}

- Square waves mutually orthogonal in time domain tested in flight on the X-48B

- Allows for estimation of individual control surface effectiveness for closed-loop flight control systems

- Care taken in limiting amplitude of aircraft response

- More precise than temporal separation method

- Not sensitive to frequency of the input within the range tested

- 20 seconds sufficient for most applications 
